\title{
An Earth Observation Land Data Assimilation System (EO-LDAS)
}

Article

Accepted Version

Lewis, P., Gomez-Dans, J., Kaminski, T., Settle, J., Quaife, T., Gobron, N., Styles, J. and Berger, M. (2012) An Earth Observation Land Data Assimilation System (EO-LDAS). Remote Sensing of Environment, 120. pp. 219-235. ISSN 0034-4257 doi: https://doi.org/10.1016/j.rse.2011.12.027 Available at https://centaur.reading.ac.uk/28468/

It is advisable to refer to the publisher's version if you intend to cite from the work. See Guidance on citing.

To link to this article DOI: http://dx.doi.org/10.1016/j.rse.2011.12.027

Publisher: Elsevier

All outputs in CentAUR are protected by Intellectual Property Rights law, including copyright law. Copyright and IPR is retained by the creators or other copyright holders. Terms and conditions for use of this material are defined in the End User Agreement.

\section{www.reading.ac.uk/centaur}

\section{CentAUR}

Central Archive at the University of Reading 
Reading's research outputs online 


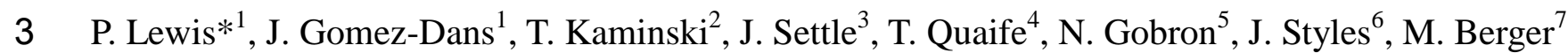

5 1. Department of Geography, UCL, and National Centre for Earth Observation, Gower St., London, 6 WC1E 6BT, UK.

7 2. FastOpt, Lerchenstr. 28a, D-20767 Hamburg, Germany.

8 3. National Centre for Earth Observation, University of Reading, Reading RG6 6AL, UK

9 4. College of Life and Environmental Sciences, University of Exeter and National Centre for Earth 10 Observation, Peter Lanyon Building, Penryn, Cornwall, TR10 9EZ, UK.

11 5. European Commission, DG Joint Research Centre, Institute for Environment and Sustainability, 12 Global Environment Monitoring Unit, TP 272, via Enrico Fermi 2749, I-21027 Ispra (VA), Italy.

13 6. Assimila Ltd., 1 Earley Gate, Reading RG6 6AT, UK.

14 7. ESA ESRIN, Science Strategy, Coordination and Planning Office (EOP-SA), Via Galileo Galilei, 15 Casella Postale 64, 00044 Frascati (RM), Italy. medium to moderate-resolution optical constellations, Leaf Area Index, Chlorophyll

\section{Abstract}

21 Current methods for estimating vegetation parameters are generally sub-optimal in the way they exploit information and do not generally track uncertainties. We look forward in the future to operational data assimilation schemes to track land surface processes and exploit multiple types of observation. Data assimilation schemes seek to combine observations and models in a statistically optimal way taking into account uncertainty in both, but have not yet been much exploited in this area. The EO-LDAS scheme and prototype, developed under ESA funding is designed to exploit the 
27 anticipated wealth of data that will be available under GMES missions such as the Sentinel family

28 of satellites to provide improved mapping of land surface biophysical parameters. This paper

29 describes the EO-LDAS implementation, and explores some of its core functionality. EO-LDAS is a

30 weak constraint variational data assimilation system. The prototype provides a mechanism for

31 constraint based on a prior estimate of the state vector, a linear dynamic model, and Earth

32 Observation data (top of canopy reflectance here). The observation operator is a non-linear optical

33 radiative transfer model for a vegetation canopy with a soil lower boundary, operating over the

34 range 400 to $2500 \mathrm{~nm}$. Adjoint codes for all model and operator components are provided in the 35 prototype by automatic differentiation of the computer codes.

37 In this paper, EO-LDAS is applied to the problem of estimating a subset of six of the parameters 38 controlling the radiative transfer operator over the course of a year (> 2000 state vector elements).

39 Zero and first order process model constraints are implemented and explored as the dynamic model.

40 The assimilation estimates all state vector elements simultaneously. This is performed in the context

41 of a typical Sentinel-2 MSI operating scenario, using synthetic MSI observations simulated with the 42 observation operator, with uncertainties typical of those achieved by optical sensors supposed for 43 the data.

45 The experiments consider a baseline state vector estimation case where dynamic constraints are 46 applied, and assess the impact of dynamic constraints on the a posteriori uncertainties. The results 47 demonstrate that reductions in uncertainty by a factor of up to two might be obtained by applying 48 the sorts of dynamic constraints used here. The hyperparameter (dynamic model uncertainty) 49 required to control the assimilation are estimated by a cross-validation exercise. The result of the 50 assimilation is seen to be robust to missing observations with quite large data gaps. 


\section{$53 \quad 1.1$ Background}

54 One of the primary goals of Earth Observation (EO) is to provide objective and reliable information on the current and (particularly within the satellite EO era) historical state and dynamics of the Earth environment. A major component of this that has been a significant focus of research efforts on monitoring terrestrial vegetation, but EO data are usually of a radiometric nature and do not give direct estimates of the properties of the Earth land surface that we wish to map. Some level of inference is therefore needed.

61 Early studies in terrestrial vegetation monitoring from EO (Richardson and Wiegand, 1977; Tucker, 1979) found that simple transformations of multispectral measurements at red and near infrared wavelengths gave a signal that was responsive to the relative amount of green biomass and that

64 could be used to track vegetation dynamics (Goward et al., 1985). The attractions of such 'Vegetation Indices' (VIs) are obvious: they are visually impressive as spatial and temporal datasets;

67 the extraneous factors that can otherwise complicate lower level EO signals; and they can often 68 provide effective information for time series analyses, where the timing, rather than the magnitude 69 of events is of importance (e.g. vegetation phenology). Further, such indices can be directly targeted 70 at particular functional or physical vegetation properties, such as the fraction of absorbed 71 photosynthetically active radiation (fAPAR) or Leaf Area Index (LAI), by design (Gobron et al., 72 2002, 2010) or empirically (Rochdi and Fernandes, 2010). In the former case a calibration is 73 achieved using a set of radiative transfer model runs over a range of conditions (Gobron et al.,

74 2000). In the latter, extensive ground-based measurements must be made (Chen et al., 2002) and the 75 form of the relationship with a particular VI assumed. Such efforts are fast to process and often 76 effective, especially for near-real-time survey. They have a range of known failings (Baret and 77 Guyot, 1991), but some of these, such as dependence on the angular conditions of data acquisition 
can be reduced by treating the data to normalise for such effects (e.g. Rochdi and Fernandes, 2010).

79 Ultimately though, however much care is taken to treat such effects, methods assuming such fixed

80 mappings from VIs with 'statistical' models are open to many criticisms, some of the more

81 significant of which could be considered: (i) they fail to make full use of the information content of

82 the observational data; (ii) they (often) fail to make use of our understanding of the physics of the

83 situation; (iii) they need recalibration if conditions change (e.g. sensor band pass functions or scale

84 of observation); (iv) they tend not to treat uncertainty in the mapped product in any rigorous way

85 (mostly, they fail to consider this at all).This is a judgement call.

86

87 An alternative stratagem has been to build mathematical models of the physics of radiation

88 interactions with vegetation canopies and the intervening atmosphere, phrased as functions of

89 'control' variables (polarisation, wavebands, viewing and illumination angles etc.) and (bio) physical

90 parameters or 'state variables' (LAI, leaf chlorophyll concentration etc. for the canopy, and aerosol

91 optical depth, ozone concentration etc. for the atmosphere), and to use these to attempt to interpret

92 the satellite signal. We may call these radiative transfer (RT) models. To tie in with discussions

93 below and to provide consistency with the data assimilation literature, such models are called here

94 'observation operators' (denoted $H(x)$ ) in that they map from the state variable vector $x$ to the EO

95 signal (as a vector) $R$ for a given set of control variables, so the modelled signal vector $R=H(x)$.

96 The 'remote sensing inverse problem' then is to obtain an estimate of some function of $x, F(x)$

97 from measurements $R$. How this may be achieved is discussed in more detail below.

99 Much effort has been devoted to producing information from EO data about specific biophysical 100 quantities that are relevant to science and society. A major focus of this has been to attempt to 101 provide estimates of (green) LAI. Garrigues et al. (2008) consider four representative EO-derived global LAI products, with core spatial resolutions of $1 \mathrm{~km}$ or coarser, that use what might be 
considered state of the art methods for multi-year dataset generation. The reader is referred to that paper for detailed information on the products, a product inter-comparison and validation against independent ground measurements. The temporal resolution of the products varies from 8 days to 1 month. Three of the products (ECOCLIMAP, GLOBCARBON (V1), and CCRS) are derived from assumed VI relationships with LAI. A fourth (MODIS (C4)) uses such a relationship for a backup algorithm. Three of the products (GLOBCARBON, CYCLOPES (V3.1) and MODIS) make use of RT models in attempting to estimate the LAI. In the case of GLOBCARBON the RT model is used to calibrate the VI-LAI relationship. For MODIS a look up table derived from the RT model is used to map red and near infrared (NIR) bidirectional reflectance data to LAI, and for CYCLOPES a neural network derived from an RT model is used for the mapping from red, NIR and shortwave infrared (SWIR) portions of the electromagnetic spectrum. A feature of these uses of RT models is that they can map many channels of input data to one (or many) outputs. The one-to-one mapping used in VI design and/or calibration is then just the simplest case of this more general RT approach.

A major new effort in satellite data provision is the GMES (Global Monitoring for Environment and Security; www.gmes.info) programme (Council of the European Union, 2010). It is an EU initiative set up to provide timely information on key environmental variables for policy makers and public authorities, and is intended to be a major EU contribution to understanding and managing climate change. Six thematic areas are being developed: marine, land, atmosphere, emergency and security and climate change. The land monitoring service is provided via the GEOLAND2 project (www.gmes-geoland.info), which oversees the generation of products derived from satellite data, providing information on a wide range of variables including LAI. GMES is a European contribution to GEOSS, the Global Earth Observing System of Systems (European Commission, n.d.). The Sentinels are a series of satellites being developed by the European Space Agency that are specifically designed to address the space observation requirements of GMES. There are five Sentinel missions, each of which will consist of a pair of satellites (for details see Aschbacher et al., 
2012 and dedicated Sentinel mission papers, all this RSE issue). This paper is primarily concerned

130 with methods for the retrieval of biophysical parameters of terrestrial ecosystems, including LAI,

131 from instruments at arbitrary spatial resolutions, sun-sensor geometries and optical wavelengths.

132 Consequently the techniques described here are directly relevant to Sentinels 2 and 3 missions.

133 Sentinel 2 has a medium resolution multispectral imager (MSI) in the optical domain with 4 bands 134 at a $10 \mathrm{~m}$ resolution, 6 bands at $20 \mathrm{~m}$ and 3 bands at $60 \mathrm{~m}$. These 13 spectral channels (Table 1 ) are 135 distributed in the visible and near infrared and shortwave infrared regions. The Ocean Land Color 136 Instrument (OLCI) instrument on board the Sentinel 3 platform is a coarser (circa 500m) resolution 137 instrument, similar to MERIS that is designed for global monitoring applications. In principle the 138 system described in this paper could also be extended to other wavelength domains and 139 consequently be used to integrate data from the entire suite of EO missions.

\section{TABLE 1 ABOUT HERE}

143 An additional context for this paper is the growing interest in the application of wider constellations 144 of satellites for environmental and disaster monitoring. A manifestation of this is the NASA A-train 145 (NASA, 2010), which is a formation of complementary satellites and sensors taking observations at 146 close to the same time. Other examples include relatively low cost satellites and instruments with a 147 suite of similar instruments flying in formation to provide global daily viewing opportunities at 148 mid-resolution (10-30m), for example the Disaster Monitoring Constellation (DMC) (DMCII, 149 2010). The concept can potentially be applied to more heterogeneous systems, such as the 'virtual 150 constellation' for Land Surface Imaging (LSI) concept promoted by the Committee on Earth 151 Observation Satellites (CEOS) to optimise benefits from land remote sensing systems (CEOS, 152 2011a). There are clear benefits for monitoring frequency if data from a wider range of sensors are 153 available, but the more heterogeneous the set of sensors (in terms of spatial resolution and 154 wavelength domains) the more important it is to formalize appropriate methods to optimally merge 
information from these sources.

\subsection{Optimal estimation}

158 The remote sensing inverse problem described above can be phrased as an optimal estimation 159 problem, requiring an estimate of a distribution around the minimum of some function of an 160 observation residual vector, such as an $\ell^{2}$-norm. Our assimilation system is based on the joint 161 inversion approach of (Tarantola, 2005) and is most conveniently formulated in what is often called 162 a Bayesian context (Enting, 2002), which means that each piece of information (including any prior 163 information on the state variables) is represented by a probability density function (PDF). 164 Combining this information yields an a posteriori PDF for the parameters, which is the 165 result/solution of the assimilation problem. If all of these PDFs are Gaussian and the models 166 involved not too non-linear (potentially after a transformation) then the posterior parameter PDF 167 can also be approximated by a Gaussian:

$\rho(x)=\exp (-J(x))$

171 which is the maximum likelihood estimate of the state variables $x$, thus the minimum of a cost 172 function which takes the form:

176 where $J_{i}(x)$ is a cost function expressing a constraint $i$, a member of some set of constraints.

178 Much of the earlier literature on estimating $x$ for vegetation monitoring from a physical basis 179 concentrated on exploring options in numerical minimisation approaches (see e.g. the review by 
Kimes et al. (2000)) based almost entirely on using a single cost function $J_{o b s}(x)$ expressing a mismatch between EO data and the prediction of an observation operator $H(x)$ (a radiative transfer model). The optimisation methods explored include, but are not limited to, downhill simplex (Privette et al., 1994), gradient methods (Gill et al., 1981; Liang and Strahler, 2002), neural networks, look-up tables and genetic algorithms (GA) (Combal et al., 2003; Myneni et al., 1995; Weiss et al., 2000). Although appropriate optimisation strategies and computer implementations have been around for some time that make use of $J_{o b s}$, the gradient of $J_{o b s}$ with respect to $x$, in locating the minimum, they have not been widely used in terrestrial EO monitoring, primarily because of the perceived computational cost and numerical issues if finite difference methods are used to estimate $J_{o b s}$, and more particularly because it is no trivial job to differentiate radiative transfer models. The advent of automatic differentiation (AD) methods and tools such as TAF (e.g.

191 Giering and Kaminski, 1998, Lavergne et al., 20076) or TAPENADE (e.g. Qin et al., 2007) means that calculating $J_{o b s}$ for radiative transfer or other models is now quite feasible at computational costs not greatly dissimilar to the calculation of $J_{o b s}$. The approach has first been applied to rather simple RT models such as RPV (Lavergne et al., 2007) and a two-stream model (Pinty et al., 2007; Clerici et al., 2010), but this is equally appropriate for more complex models as we show here. The ability to make rapid, exact calculation of the gradient vector not only widens the choice of 197 algorithms that might be used to minimise the cost function, but also provides a route for potentially 198 faster state vector estimation, and perhaps most importantly allows larger dimensioned problems to 199 be tackled. Qin et al. (2008) were perhaps the first to apply AD to more complex RT models 200 (MCRM of Kuusk (1995)) using a combination of GA and a cost function-based method using $J_{o b s}$ 201 in the region of a trust region derived from the GA. In this case 7 members of the (dimension 14) state vector are estimated, but only at a single point in time. Results are not shown for parameters other than LAI, and no detailed consideration of uncertainty is included, but the ability to use AD in such scenarios is clearly demonstrated. 
Data producers and users generally have little influence over control variables to the estimation

207 problem, as satellite sensors and missions are usually designed to serve (or are used to serve)

208 multiple purposes, and involve compromises in sensor design and orbits. Any one sensor (and the 209 resultant set of control variables entailed) then will tend to be sub-optimal for a task as specific as 210 vegetation monitoring. Inevitably this results in individual EO data sources having information 211 content that is too low to provide accurate retrievals of the entire state vector space. Some 212 parameters may never be completely retrievable on the basis of observation alone, especially where 213 there is equifinality between two or more parameters over the domain of the observed data, that is, 214 when the same model state can be reached by different combinations of state variables. See for example (Beven, 2006) for an overview of this issue or Lewis and Disney (2007) for an attempt at 216 explaining mechanisms impacting this in canopy radiative transfer. The core of the issue is that the 217 observations only refer to a subspace of the unknown state variable space. In this case, no 218 information on some directions in state space can be gained from the observations, and their values 219 will have to be constrained using for example, prior information. Such problems are described as 220 being ill-posed. As an example, consider the often-desired goal of tracking the temporal evolution 221 of some parameter of interest such as LAI, to provide information on phenology. Inverting a model 222 on a daily basis where there may only be a small number of observations, or none at all, is typically 223 not possible as a single observation does not have enough information to constrain all of the state 224 vectors of typical radiative transfer models. This has been solved implicitly in the production of 225 many current EO data products by assuming the model parameters to be constant over some time 226 interval, and many of the ancillary parameters such as those governing leaf and soil properties are 227 simply assumed known (and fixed as is the case when using VIs). Assumptions such as temporal 228 invariance or knowledge of ancillary variables are pragmatic responses to the remote sensing 229 problem being ill-posed, but it is better if possible to seek less ad hoc methods for constraining our 230 estimate, especially if we wish to estimate uncertainty in the product. 
232 A mechanism that provides scope for dealing with such problems is the suite of tools that are

233 collectively referred to as 'Data Assimilation' (DA). There is no strict definition as to what

234 constitutes DA but it is taken here to mean the statistically optimal merging of data and models.

235 Optimality, in this sense, implies the need to take into account uncertainties in all parts of the 236 system.

\subsection{Data Assimilation}

239 Data assimilation can be seen as mechanism for combining models and data. The defining feature of 240 DA, at least by the definition provided in this paper, is that it enables the use of additional 241 assumptions to make parameter estimation viable in situations that exhibit ill-posedness. In essence, 242 we have a mechanism through equation 1 to combine multiple constraints. An example of this that 243 has long been used either explicitly or implicitly in the inference of land surface parameters from 244 EO is constraint via a priori estimates of parameter values or ranges (or more generally, 245 distributions). What DA specifically brings to bear on the problem is a dynamic model of parameter 246 evolution in space and/or time.

248 Early examples of data assimilation systems are those used to improve short-range weather 249 predictions from meteorological models (Ghil and Malanotte-Rizzoli, 1991). In these systems the 250 number of state variables is typically huge, often greater than $10^{6}$, because of the large number of 251 interconnected sub-domains used to represent the atmosphere in a 3D grid. The number of 252 observations available is typically several orders of magnitude less than this, and in consequence 253 the problem is ill-posed. However, including a constraint that the final solution should not diverge 254 too far from an a priori estimate (typically supplied by a previous model run) tends to result in a 255 tractable solution. The schemes used for these problems are referred to as 'variational', being based 256 in the field of mathematics dealing with the calculus of variations, and are closely related to the DA 257 system described in this paper. A 'strong constraint' variational DA system assumes that the 
underlying process model prescribing the state vector evolution is correct (i.e. there is a model

trajectory that matches the observations). In this case it is generally only the initial state of the system that is estimated by the DA procedure, but this approach can also be used to calibrate models (i.e., to optimise estimates of model process parameters) (Knorr et al., 2010). If the state vector is allowed to deviate from the model predictions then this is referred to as a 'weak constraint' DA system (Zupanski, 1997). It is this latter type that is used here and is discussed more completely in later sections.

We note that these systems have been exploited to estimate LAI from MODIS data by making use of a coupled phenology temporal trajectory model with a radiative transfer model (Xiao et al. 2009; Xiao et al. 2011). MODIS LAI is assimilated into a crop model using a variational technique in Fang et al. (2008a). The variational approach is shown to help in retrieving surface fluxes in Olioso et al. (2005) and Qin et al. (2007), and has found wide application in the hydrological literature (see for example McLaughlin, (2002)).

Another related set of techniques in the DA canon may be called sequential methods. The most widely-known and widely-used example of these is the Kalman Filter. Sequential methods generally only consider observations at a single time step and adjust the model state vector at that time by an amount proportional to the differences between the observations and the predictions of those observations using that model state. Using a variant of the Kalman Filter, known as the Ensemble Kalman Filter (Evensen, 2003), Quaife et al. (2008) demonstrated the assimilation of satellite reflectance data into a simple ecosystem model using an RT model as observation operator. Other efforts have used these techniques to assimilate e.g. snow data (Slater and Clark, 2009) or MODISderived LAI into a phenology model (Stöckli et al., 2008). The related technique of particle filtering has been used to assimilate microwave temperature in order to infer soil moisture dynamics in Qin et al. (2009). 
285 The Earth Observation Land Data Assimilation System (EO-LDAS) study funded by ESA aims at 286 supporting the generation of a generic land data assimilation system by using the full information 287 content provided by observations from satellite constellations. Such a system, in eventual 288 operational form, is intended primarily to improve the quality and consistency of land surface 289 products generated from multi-sensor EO data. The project is focussed on developing a generic 290 scheme and software prototyping for use with medium to mid spatial resolution (in the range $10 \mathrm{~m}-$ $291500 \mathrm{~m}$ ) optical data. The principal design concept is to allow integration of data from different 292 satellites observing the surface of the earth at different sun-sensor geometries, wavebands and 293 spatial scales, such as that supplied by Sentinels 2 and 3, in a physically consistent manner, and to 294 provide information on the state of the surfaces with well-quantified estimates of uncertainty. It also 295 demonstrates the idea that predictions based on data from one sensor can be made from a DA 296 system driven by observations from another, a concept that could potentially be used to aid 297 vicarious sensor calibration.

\section{The EO-LDAS prototype}

\subsection{The EO-LDAS Scheme}

301 The EO-LDAS prototype is an initial version of the scheme, designed to 302 carry out a core set of DA functions. In particular, in the scheme, it performs an atmospheric 303 correction of images to top-of-canopy reflectance, retrieves canopy state variables using surface 304 reflectance data and a constraint model and simulates top-of-atmosphere radiance or reflectance for 305 a given surface and atmosphere. This preserves the essential features of a more comprehensive 306 system (incorporating a fuller coupling between the surface and atmosphere), while allowing 307 development and further study of the most important elements - the observation operators and the 308 assimilation techniques. 
310 To simplify the prototype, we have assumed a large length scale for variations in atmospheric

311 scattering properties, and a very short length scale for surface variability. With these assumptions,

312 we can correct an image (or sub-image) with a single set of atmospheric state variables, use 313 reflectance data in a multi-temporal assimilation on a cell-by-cell-basis, and simulate a top-of314 atmosphere radiance field using the same atmosphere for each of a set of model grid cells. This 315 process can be iterated to achieve the surface-atmosphere coupling. To relax either constraint 316 would mean we have to deal with the inversion of a coupled surface-atmosphere problem over a 317 large number of cells, which would require considerable computing resources, both in terms of 318 memory (for the covariance structures involved) and the time needed to carry out the actual 319 inversion, without necessarily improving our ability to monitor the land surface. A tutorial guide 320 explaining the functionality and use of the prototype system is available online ${ }^{1}$.

322 The DA system can be considered to have two main components: (i) a set of constraints, expressed 323 via equation 1; (ii) an assimilation algorithm, i.e. a way to apply the constraints to achieve the 324 optimal estimate of the state vector. The set of constraints in EO-LDAS involves: (i) an 325 observational constraint $J_{o b s}(x)$, requiring data (from EO or ground measurements) and a model for 326 translating from state space to observation space (the observation operator); (ii) a dynamic model 327 constraint $J_{\text {model }}(x)$, conditioning the temporal (and/or spatial) evolution of the state vector; (iii) 328 physical or empirical bounds and/or distribution constraints $J_{\text {prior }}(x)$ to the state vector elements; 329 Thus, in EO-LDAS, equation 1 becomes:

$$
J(x)=J_{\text {obs }}(x)+J_{\text {prior }}(x)+J_{\text {model }}(x)
$$

333 Each of these constraints has associated with it an error model. In the following sections, we

\footnotetext{
${ }^{1}$ http://www2.geog.ucl.ac.uk/ plewis/eoldas/
} 
334 describe the set of constraints and the DA algorithm. We stress that in the text below, we use the

$335 \operatorname{symbol} x$ to refer to the set of state variables that we wish to estimate. In EO-LDAS this essentially 336 means a representation of the state at each sample points in time (and/or space) that we consider.

337 So, for example if we were trying to estimate Leaf Area Index and leaf Chlorophyll content at one 338 location for every day of the year, we would have a state vector with $361 \times 2$ elements. In addition, 339 EO-LDAS has the capacity to augment this state vector with 'static' state representations (some 340 term affecting one or more of the constraints that we wish to be considered constant in space/time).

\subsection{Observational Constraint}

343 Given the EO context of this system, at least one of these constraints should be based on

344 observations. The cost function is generally weighted for observation and observation operator 345 uncertainty and correlation (assumed in EO-LDAS Gaussian and described by $C_{o b s}$ ):

$$
J_{o b s}(x)=\frac{1}{2}\left(\begin{array}{ll}
R & H(x)
\end{array}\right)^{T} C_{o b s}^{1}(R \quad H(x))
$$

where ${ }^{T}$ denotes the transpose operator. This is the penalisation associated with differences between the predicted and observed reflectance values. The covariance matrix $C_{o b s}$ describes the uncertainty in the observations (and also formally, in the observation operator). As noted, the purpose of the observation operator $H(x)$ is to translate information from the state space to that of the observations, and is in practice a radiative transfer model. For ease of implementation (mainly

354 involving spectral sampling issues), when different sensor types are used in EO-LDAS, a set of $J_{o b s}(x)$ terms is developed, with one for each sensor type.

357 There have been many attempts to create observation operators $H(x)$, varying in complexity, 358 accuracy and computational cost. Goel (1988) provides a review of most of the concepts for 
radiative transfer model developed for reflectance from vegetation canopies at optical wavelengths

360 (see also (Goel and Thompson, 2000), with (Tha Paw U, 1992) covering related materials for 361 thermal emitted radiation and (Fung and Chen, 2010) for the microwave domain. Some updates and 362 model intercomparisons are provided by Sobrino et al. (2005) (thermal) and Widlowski et al., 363 (2007) (optical). The focus in this paper, and in the prototype EO-LDAS is on the use of optical 364 sensor data, but the approach outlined here is easily adapted for use in other wavelength domains.

366 In a similar way, atmospheric properties, such as aerosol optical depth and water vapour content, 367 need to be accounted to obtain accurate estimates of surface properties. This can be achieved by 368 coupling the surface model with an atmospheric model, and solving for both the surface and 369 atmosphere parameters simultaneously (Verhoef and Bach, 2003). Some (probably most) 370 approaches to surface interpretation use surface reflectance that has already been 'corrected' for 371 atmospheric effects (Vermote et al., 2002), but a full decoupling of the problem, at optical 372 wavelengths at least, cannot be achieved without knowledge of the surface Bidirectional 373 Reflectance Distribution Function (BRDF) (Lyapustin and Knyazikhin, 2001; Lyapustin et al. 2006) 374 (or at least a normalised form of this) (Vermote et al., 1997).

376 The observation operator we use in this paper is developed from the original semi-discrete model of 377 Gobron et al., (1997). It has a state vector describing canopy architecture and three spectral terms, 378 although these are all defined as functions of other parameters as described below (Table 2). The 379 soil reflectance is assumed Lambertian in the model, although it could be adapted to incorporate a 380 soil directional reflectance model. As stated here then, the (canopy-soil) model estimates the 381 directional reflectance factor at a set of viewing and illumination angles for a given narrow 382 waveband. Since the model must be capable of predicting the reflectance at arbitrary (solar 383 reflective) wavelengths, spectral models are incorporated in the code to predict the soil 384 (Lambertian) reflectance and leaf (bi-Lambertian) reflectance and transmittance. Since model 
derivatives are required, we use for simplicity here: (i) the linear soil reflectance model of Price (1990); and (ii) an approximation to the PROSPECT leaf reflectance/transmittance model of (Féret et al., 2008), being a minor modification of the model of (Jacquemoud and Baret, 1990). The approximation was developed for possible processing speed enhancements, but is identical in form to PROSPECT if the parameter $\mathrm{N}$ (table 2 ) is 1 , and very close to the original model over the range of $\mathrm{N} 0.8$ to 2.5 .

The soil spectral model of Price (1990) characterises a given soil at field capacity as a linear combination of Empirical Orthogonal Functions (EOFs) based on a database of moist (field capacity) soil spectra. Four EOFs are found to account for $99.6 \%$ of the cumulative variance of all the soils considered, so, as is usual, we use up to four terms in this implementation. Parameter ranges in Table 2 come from (Price, 1990), figures 11-13.

TABLE 2 ABOUT HERE

TABLE 3 ABOUT HERE

401 The leaf angle distribution is categorised in the model of Gobron et al., (1997) and so not set by the 402 assimilation procedure (i.e. it must be pre-defined or the different categories assessed separately: 403 this could ultimately be improved using a continuous description). The assimilation scheme can 404 provide estimates of the remaining (12) state variables for each time period modelled. Following 405 (Weiss et al., 2000) we apply approximate linearization functions to some of the terms (Table 3). 406 The reasons this is appropriate here are: (i) they better condition the problem for optimisation; (ii) 407 the assumptions of Gaussian distributions of errors are more appropriate in this case.

409 Differentiated versions of the observational cost are required to enable the use of efficient gradient 410 descent minimisation routines, so we can benefit from access to $J_{o b s}(x)$, the derivative of $J_{o b s}(x)$ 
with respect to $x$. This is:

412

413

$$
J_{o b s}(x)=H(x)^{T} C_{o b s}^{1}(R \quad H(x))
$$

where $H(x)$ is the derivative of $H(x)$ with respect to $x$. An adjoint code of the cost function for

416 the semi-discrete model, i.e. code for direct calculation of $J_{o b s}(x)$ that avoids the need for explicit 417 calculation and storage of $H(x)$, was generated from the source code of the model by the 418 automatic differentiation tool TAF (Giering and Kaminski, 1998). The adjoint code implements the 419 chain rule of differentiation in the so-called reverse mode. It provides the gradient information that 420 is accurate up to machine precision at a computational cost that is not greatly dependent of the 421 length of the gradient vector and well below that of the multiple runs of the semi-discrete model 422 that would be required for a finite difference estimate.

424 We obtain an estimate of the posterior uncertainty through consideration of the curvature at the 425 global minimum in state space. This is provided by the inverse of the sum of the constraint 426 Hessians, the Hessian for this constraint being $J_{o b s}(x)$ :

$$
J_{o b s}(x)=H(x)^{T} C_{o b s}^{1} H(x) \quad H(x)^{T} C_{o b s}^{1}(R \quad H(x))
$$

430 Although it should be possible to develop a Hessian code in much the same way as done for the first 431 derivative, that has not yet been done within EOLDAS, so a linear approximation to the Hessian is 432 achieved, using finite differences. As we will see below, the algorithm used to perform the 433 optimisation is iterative, but the potentially high cost of using finite differences for the Hessian is 434 unimportant in this sense, as it only has a role in estimating the posterior uncertainties. 


\subsection{Process model constraint}

437 The EO-LDAS prototype is designed to allow the user to interface their own constraints, so long as

438 they provide code to calculate the cost function and its first and second order derivatives. This 439 allows a mechanism whereby (bio)physical process models can be used to constrain the solution 440 and or estimates of the variables controlling those models can be developed. The focus of the 441 prototype software and that of this paper are on understanding how to use DA concepts to improve 442 estimates of biophysical variables from EO data, rather than to test specific process models 443 however. For this reason, we have currently only implemented a linear process model in the system:

$M(x)=A x+b$

447 where $A$ and $b$ are a matrix and vector respectively. One advantage of designing the prototype 448 system in this manner is that it provides a flexible framework for changing the underlying model. 449 Unlike in a sequential system, this formulation directly allows for any model state vector element to 450 be linked to any other, since $x$ here contains the state representation at all sample times (spaces), so 451 different time/space scales can be readily incorporated. The cost function associated with this 452 process model then is:

$J_{\text {model }}(x)=\frac{1}{2}\left(\begin{array}{ll}x & M(x)\end{array}\right)^{T} C_{\text {model }}^{1}\left(\begin{array}{ll}x & M(x))\end{array}\right) \frac{1}{2}\left(\left(\begin{array}{lll}I & A\end{array}\right) x \quad b\right)^{T} C_{\text {model }}^{1}\left(\begin{array}{lll}I & A) x & b\end{array}\right)$

where $I$ is the identity operator. $J_{\text {model }}(x)$ is the cost incurred by departure of the model state from that predicted by an underlying process model. An interpretation of $A$ is as the model derivative.

458 The model uncertainty matrix $C_{\text {model }}$ therefore expresses the uncertainty in this derivative, including 459 any inherent uncertainty in the process model. It such a case, it might often be pragmatic to specify 460 only diagonal terms in $C_{\text {model }}$ as further details of model structure are often difficult to obtain. In 
any case, we can see that EO-LDAS could be interfaced to a process model such as the Carbon Flux

462

463

464

465

466

467

468

469

470

471

472

473

474

475

476

477

478

479

480

481

482

483

model DALEC used by Quaife et al. (2008) or any other for which the derivative might be obtained (e.g. using $\mathrm{AD}$ ) by augmenting the state vector $x$ by any terms that we might wish to drive the model.

Whilst the EO-LDAS scheme allows for linking to 'biophysical' or other process models, that is not the main focus of the prototype. Indeed, there are many cases, for instance when conducting a comparison of information derived from EO data and some biophysical model trajectory, when it may be undesirable to directly incorporate a detailed process model. Further, and perhaps more importantly, a fundamental requirement of the EO-LDAS system is that the state vector, $x$, contains at least the parameters of the observation operator $H(x)$ for every point in time (and/or space), and many of these may not be provided by a biophysical process model designed, for example, to estimate total Carbon fluxes. We should see the matrix $A$ (and if needed, the vector $b$ ) then as a much more general interface to 'process modelling' within an optimal estimation environment.

We can for example consider the benefits of approaches such as Twomey-Tikhonov regularisation or variations around this theme (Rodgers, 2000). Examples of this that we explore further below are first and second order difference constraints. In essence these improve the conditioning of the inverse problem by smoothing or regularising the solution, which comes about because they constrain derivatives (first or second order here) to be zero. In a weak constraint DA system such as that used here, the model is not strictly enforced (this would be clearly undesirable in these derivative constraints) but rather the degree of smoothness in the outcome is traded off against the other factors in $J(x)$ through the model uncertainty matrix. In other words, the cost function will penalise temporal trajectories of parameters that are not flat, but this is 'balanced' with a goodness of fit to the observations and departure from the prior estimate. In practice this constrains the solution toward a smooth evolution by minimising the high frequency components of the temporal 
parameter trajectory. A similar approach has been taken by Quaife and Lewis (2010) for linear

488 observation operators. Viewing this form of solution as a combination of state variable estimation 489 and filtering, we note that the filter characteristics are controlled by the nature of matrices $A$ and $490 C_{\text {model }}$, the former controlling the cut-off frequency of the filter and the latter, if simply diagonal, 491 controlling the degree of dampening of the unwanted high frequencies. In this context, we can consider $b$ a bias term, which we set to zero. In this case:

$J_{\text {model }}(x)=\frac{1}{2}(D x)^{T} C_{\text {model }}^{1}(D x)$

where $D=\left(\begin{array}{ll}I & A\end{array}\right)$. The derivatives of this are: $J_{\text {model }}(x)=D^{T} C_{\text {model }}^{1} D x$ and $J_{\text {model }}(x)=D^{T} C_{\text {model }}{ }^{1} D$. To achieve Twomey-Tikhonov regularisation then, which we view as an empirical process model, $D$ here becomes simply a $\left(\mathrm{N}^{\text {th }}\right.$ order) differential operator (Quaife and Lewis, 2010). In many situations, we must assume the uncertainty in this empirical constraint unknown. The minimum error model then is a constant value for which we can use a scalar term :

$$
J_{\text {model }}(x)=\frac{2}{2} x^{T}\left(D^{T} D\right) x
$$

504 We can interpret as a 'smoothness term (or ${ }^{1}$ as a roughness term) that controls the weighting 505 of the derivative (model) constraint with respect to the other constraints. It is worthwhile at this 506 point trying to relate this back to the discussions on process models. This is most readily achieved 507 by considering a first order derivative constraint. If applied at lag 1 day for a temporal constraint, 508 we can interpret this as an expectation that the state vector tomorrow will be the same as today (i.e. 509 the derivative is zero). If we want to relate this to equivalent sequential methods, we can say that 510 this is a zero-order process model. The term ${ }^{1}$ then can be interpreted as uncertainty (phrased as 
511 standard deviation) in this model, or alternatively as the growth in uncertainty over a one day

512 period. Similar interpretations apply for other derivative constraints: a second order derivative

513 constraint is equivalent to a first order process model. Equation 5 then is a viable empirical process

514 model constraint, but we have yet to tackle the fact that the smoothness is unknown. We also note

515 that if we use a scalar for , we are assuming the same smoothness for all state variables at all times 516 (places).

518 An option that arises with dynamic models (where we are making connections between elements of 519 the state vector at different times (places) is what to do about boundary conditions. Even with a 520 simple differential model this needs consideration in forming the $D$ matrix. Among the various 521 options, especially when dealing with annual or multi-annual datasets, an attractive one is to assume 522 periodic boundary conditions, and that is done here. This means that in calculating $D$ at the end of 523 the year (edge of the matrix) we perform the digital differential with state elements from the 524 beginning of the year.

526 It is generally found (e.g. Twomey (2002)) that quite a broad range of model uncertainty 527 (smoothness) estimates can provide an acceptable solution, so we do not expect the results to be 528 overly-sensitive to the choice of this 'hyper-parameter'. We could make a rough guess at the model 529 uncertainty, but that is likely to be unsatisfactory in the general case. If we under-estimate it by too 530 much, we can over-dampen most of the state vector. Equally, if we greatly over-estimate the model 531 uncertainty, the impact of the temporal constraints is minimal: in the extreme, an infinite model 532 uncertainty (zero smoothness) leads to a solution without model constraint. Whilst there are several 533 strategies that can be employed to estimate the model uncertainties (hyper-parameters), perhaps the 534 most fruitful in the context of EO-LDAS is running a cross-validation exercise. The idea is that an 535 independent dataset is used to test the robustness of the solution for a particular value of the hyperparameters. An optimal estimate of the hyper-parameters (or distribution thereof) can be obtained 
by minimising a cost function with the independent observations. This can be achieved with a subset of observations to test a solution obtained from the rest of the dataset, a strategy that when repeated over different subsets becomes known as generalised cross validation becomes known as generalised cross validation (Wahba, 1990, Eilers, 2003 and Lubansky et al., 2006).. Alternatively,

541 we might use data from an independent sensor, although accurate absolute calibration between the 542 sensors is needed for that.

\subsection{Prior Constraint}

545 An additional constraint mechanism is implemented in EO-LDAS, that we term a prior constraint.

546 Its role, via the cost function $J_{\text {prior }}(x)$ is to impose a penalty for deviation from some previously 547 defined state, $x_{\text {prior }}$ :

$$
J_{\text {prior }}(x)=\frac{1}{2}\left(\begin{array}{ll}
x & x_{\text {prior }}
\end{array}\right)^{T} C_{\text {prior }}^{1}\left(\begin{array}{ll}
x & x_{\text {prior }}
\end{array}\right)
$$

where $C_{\text {prior }}$ expresses the uncertainty of the prior model state, a measure of our belief in the prior 552

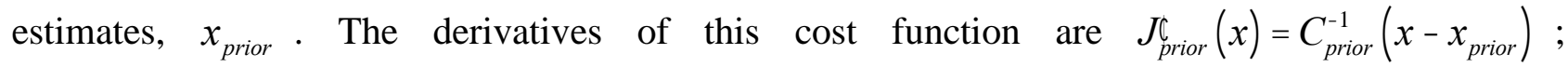
$J_{\text {prior }}(x)=C_{\text {prior }}^{1}$. A comparison of equations 6 and 8 shows that this is really just another form of model constraint, with $M(x)=x_{\text {prior }}$, which can be achieved with the existing model constraint by setting $b=x_{\text {prior }}$. In practice, this allows us to enforce a prior belief in the distribution and range of the state vector elements (e.g. a climatology or physical or otherwise know 'reasonable' 557 distributions), although only Gaussian distributions can be considered.

The various constraints discussed above provide the cost function in equation 1 through their 
summation. This also applies to the derivatives $J(x)$ and $J(x)$. The cost function $J(x)$ is minimised using a gradient descent method (i.e. using $J(x)$ ). Bounds are applied as a final constraint to the solution, to ensure that the state vector remains within physical limits. These can be 564 altered by the user for any particular run of the system via a configuration file or command line interface. In the EO-LDAS prototype we use the limited memory Broyden-Fletcher-GoldfarbShanno (L-BFGS-B) algorithm described in (Byrd et al., 1995; Zhu et al., 1997). In principle, 567 however, a number of different gradient descent algorithms could be used. The L-BFGS-B was selected for its efficient memory handling for high dimensional problems and the fact that it can

571 The algorithm then is quite straightforward: (i) read in configuration information and observations;

572 (ii) provide an initial estimate of all state vector elements that we wish to estimate; (iii) iterate 573 within the optimisation routine until convergence is reached (or using other criteria) to estimate the 574 state vector; (iv) calculate the Hessian and then its inverse to provide the posterior covariance 575 matrix, the estimate of uncertainty.

577 It is instructive to consider the contribution of these three terms in the estimates of Hessian matrix. 578 The observational term can be ill-conditioned if the observations exhibit little sensitivity to some or 579 all of the state variables, for example due to poor combinations of spectral and/or angular sampling. 580 The addition of the prior and dynamic model terms then results in improved conditioning of $J(x)$, 581 as these extra terms compensate for the lack of observation sensitivity to some of the state variables. 582 They also provide the ability to interpolate (i.e. rely more on the process model) between where we 583 have observations. Importantly, the uncertainties are tracked throughout this process, so when e.g. 584 interpolating over large gaps, we get the expected increase in uncertainty.

586 The DA system developed here can be viewed an extension of the methodologies that have been 
applied to inverting radiative transfer models by minimising a cost function. The addition of a linear

588 dynamic model therefore only adds a handful of extra parameters to the problem (namely, the nature

589 of the dynamic model itself and the associated covariance matrix, $C_{\text {model }}$, which may be simply

590 diagonal). This is in a marked contrast with similar methodologies that either use a long time series

591 of data for inverting one single parameter (in the case of inverting LAI as in (Fang et al. 2008b;

592 Xiao et al. 2009; Xiao et al. 2011). The temporal smoothness constraint is in itself an important

593 feature, which is usually performed as a post-processing step after the parameter retrieval (Lu et al. 594 2007).

\section{Experimentation}

597 We present a series of experiments to demonstrate the operation of the EO-LDAS prototype and to explore the sorts of capabilities such a system could provide with data from the Sentinel-2 MSI sensor (see table 1a for waveband information for Sentinel-2 MSI). The experiments use synthetic data for observations i.e. are derived from running the observation operator for a given state vector

601 for what we suppose to be typical Sentinel-2 scenarios over one calendar year. We simulate top hat 602 function bandpass functions (1 nm sampling) according to the information in Table 1 (see also 603 Drusch et al., 2012, this issue). The main aim of the experiments is to determine the improvement, 604 in terms of reduced uncertainty, in biophysical parameter estimation that might be obtained by 605 applying the EO-LDAS prototype for such scenarios. A subsidiary aim is to demonstrate the 606 capability of the DA system to make predictions of data from a sensor not used in the DA process. 607 Here, we do this by using the state vector estimates derived from the DA with synthetic MSI data, 608 and make predictions of what a SPOT-5-like instrument would view (described below). These data 609 are used in a cross-validation exercise within the experiments.

\section{3.1. Experimental setup}

612 In these experiments, we control the time trajectory of a subset of model parameters according to 
613 the functions given in Table 4, where $t$ is the relative day of year (DOY) i.e. DOY normalised by

614 365. All other parameters take their default values given in Table 2. The functions for LAI and 615 chlorophyll broadly mimic typical trajectories of these terms for crops: for LAI, a flat initial period, 616 followed by a rise to maximum LAI and then a symmetric decrease; for Chlorophyll, a linear rise 617 and decrease. The more arbitrary functions used for the soil brightness term $\mathrm{s}_{1}$ we include to mimic 618 rather broad variations over the year that might be supposed to be responses to soil moisture. A 619 similar function is used here for leaf water, with a time lag of 36.5 days. The quite large variation of 620 these two latter terms is intended primarily to allow the operation of the data assimilation scheme to 621 be explored over a wide range of conditions, rather than to too closely mimic some particular 622 situation. In that context, the rather large time lag between soil brightness variation and leaf water 623 content is unrealistic, but a larger phase between these terms should test the system to a greater 624 extent than having all parameters following similar trajectories. Although the full set of state vector 625 elements is 13 for each time sample, we attempt to retrieve only the 6 elements (no 1, 4, 6, 7, 8, 9 in 626 table 2) (per time sample) that we vary in these experiments, i.e. we assume the remaining elements 627 fixed and known. This is partly to reduce the computational time required for the DA and more 628 broadly because we believe it is sufficient to demonstrate the principles underlying the DA method. 629 It is quite feasible to permit an estimation of 12 of the 13 elements (not the categorical variable 630 directly through this method) but this is not the purpose of this exercise, and (arbitrary) variations in 631 these additional terms would need to be defined to achieve this.

TABLE 4 ABOUT HERE

635 To approximate the Sentinel-2 MSI acquisition geometry (ESA, 2010), we assume one sample every 5 days (73 samples over the year), with a solar zenith angle corresponding to 10:30 local time at $50^{\circ} \mathrm{N}$, random relative azimuth and random view zenith between $0^{\circ}$ and $15^{\circ}$. Whilst these 
close enough to develop an understanding of the likely behaviour of the data. The random azimuth,

640 for example, is clearly in error, but since the view zenith angle is so restricted, this will have very

641 little impact; the local time at $50^{\circ} \mathrm{N}$ will in reality be slightly later than the nominal equatorial 642 crossing time used here, but the details of the solar zenith angle are less important here than 643 inducing a typical variation over the year $\left(32^{\circ}\right.$ to $76^{\circ}$ here). The simulation of one sample every 5 644 days mimics close to the maximum sampling achievable by MSI on 2 Sentinel platforms.

646 Synthetic observations were also generated for a SPOT5 HRG-like instrument. This sensor has four wavebands (500-590, 610-680, 790-890 and 1530-1750 nm) (CEOS, 2011b). We have assumed a revisit period of 13 days (to be out of sync with the synthetic MSI observations), although the differences are only up to two days from the MSI observations. The view zenith angle was limited to $+/-25$ degrees from nadir, with a random azimuth and a local overpass time of 10.30 . In total, 28 observations were available in this dataset.

653 Uncorrelated Gaussian noise is added to the observations as part of the data synthesis. We assume 654 the standard deviation of this to vary linearly from 0.008 at the shortest wavelength to 0.020 at the 655 longest, for both the MSI and SPOT-5 HRG. These values are broadly twice those claimed for 656 atmospheric correction of data from the NASA MODIS instrument (Roy et al., 2005). If an 657 atmospheric 'correction' were performed on the data, we would generally expect the uncertainty in 658 surface reflectance to be correlated across wavelengths, as e.g. an under-estimation of aerosol 659 optical thickness would likely give rise to an over-estimate in reflectance for the shorter wavelength 660 bands. Here, we have inflated the assumed (MODIS) uncertainties by a factor of two to take some 661 account of such likely correlation. This highlights one of the benefits of ultimately using a more 662 fully coupled surface-atmosphere observation operator, in that such features would fall naturally out 663 of the model formulation and random noise might be more reasonably assumed for top of 664 atmosphere radiance or reflectance. However, for the purposes of these experiments it is sufficient 
to treat only the surface (canopy-soil) elements of the observation operator.

667 We term this simulation set 'complete' for the purposes of this paper, in that it expresses a rather 668 idealised situation where no clouds are present. A second synthetic observation set that we term 669 'cloudy' (36 observations for MSI and 15 for SPOT-5 HRG) is derived from this, for which we have 670 removed $50 \%$ of the observations according to a correlated random function to mimic persistence of 671 cloud cover. This induces ('cloud') data gaps of up to 60 days (mean gap 10.3 days, standard 672 deviation 12.6 days for MSI).

673

674 As noted above, the cost function minimisation is achieved in EO-LDAS with the L-BFGS-B 675 algorithm. A bounded minimisation is performed within this code, with the limits specified on the 676 (transformed) state variables given in Table 2 (transformations in Table 3). Thus, all state variable 677 estimations below proceed with the prior knowledge of an upper and lower bound. There are several 678 convergence criteria that can be used with the L-BFGS-B, including an absolute threshold on the 679 cost function and a relative (per iteration) threshold. In all experiments, these are set to low values, 680 which means that more iterations might be employed than strictly necessary in any operational 681 context, but making sure that the global minimum (or very close to it) is reached in each estimation.

682 Because of the additional costs of processing full bandpass functions, all 'initial' processing is 683 performed using the median $(1 \mathrm{~nm})$ wavelength of each waveband. A 'polishing' step is then 684 performed to achieve convergence from this starting value, using the full bandpass sampling. The 685 effect of applying the full bandpass functions tends to be generally quite minor.

687 We have initially tested the system without observational noise and confirm that the scheme 688 retrieves the truth to within the bounds implied by the convergence criteria and machine precision. 689 Processing time for a single set of 73 time samples with MSI spectral sampling, solving for 6 state 690 vector elements for each day of the year (2190 in total), is currently several hours on a $3 \mathrm{GHz}$ Intel 
processor on a single core, but this is partially due to very stringent convergence criteria used whilst testing the code and partially because this prototype implementation requires some significant 693 efforts in computer code optimisation.

In all experiments, we set the prior estimate of the state vector to the values shown in table 2, with very large diagonal uncertainty terms (8). This effectively removes the prior constraint from 697 consideration in these experiments, as we wish to conduct experiments based only on model and 698 observational constraints here.

In the following sections, we examine the result of applying the weak constraint variational data 701 assimilation approach described above to the synthetic dataset. For all cases, we assume that the 702 uncertainty in the observations is known and that it is Gaussian and uncorrelated between 703 wavebands and between dates. In the first case (3.2), we solve for state vector estimates assuming 704 no dynamic model constraint other than the weak prior (standard deviation 8). This acts as a 705 baseline for further experiments. In the second case (3.3) we assume that model uncertainty is 706 unknown and attempt to solve for it and the state vector for each day in the year with a form of 707 cross-validation exercise using the SPOT-5 HRV synthetic observations. The 'true' values of for 708 individual state vector elements are shown in Table 5. The DA is performed with the 'complete' (i.e. 709 5-day sampling MSI) dataset in that case. Finally, we repeat that experiment for the 'cloudy' dataset 710 (3.4).

TABLE 5 ABOUT HERE

714 Graphical results (figures 1-2) are presented as untransformed biophysical variables (i.e. LAI, $\mathrm{C}_{\mathrm{ab}}$, $715 \mathrm{C}_{\mathrm{w}}, \mathrm{C}_{\mathrm{dm}}, \mathrm{N}$ and $\mathrm{s}_{1}$ ), showing: the 'true' ('original') state vector (dashed line); circles and error bars 716 (shaded region) shows mean and 95\% credible interval bounds (at plus/minus 1.96 standard 
717 deviations). We will term 1.96 standard deviations 'uncertainty' for the remainder of the paper,

718 unless the statement is otherwise qualified. The uncertainty bounds are slightly larger for the upper

719 limits than for the lower limits (other than for $\mathrm{N}$ and $\mathrm{s}_{1}$ ) due to the nature of the transformations 720 used in the approximate linearization (table 3). Tabular results for the experiments (tables 9-12) are 721 expressed in transformed parameter space, as that is the space in which the state vector is inferred 722 and in which the Gaussian statistics derived are most natural.

\section{$724 \quad 3.2$ Baseline estimates}

725 We first produce a baseline estimate of the six state variables over the 73 time periods in the year, assuming no constraint to the solution other than the bounds noted above, the (noisy) observations,

727 knowledge of the uncertainty in the observations, and the weak prior constraint.

729 The results for the baseline experiment are produced using the EO-LDAS system with each 730 observation set (i.e. all wavebands, but only one angular sample) independently. The algorithm 731 requires an initial guess of the state vector and iterates to its final estimate. The initial estimate of 732 the state vector in each case and all subsequent estimates is taken as the value used in the prior 733 constraint.

FIGURE 1 ABOUT HERE

737 In figure 1, the column titled 'single obs inversion' shows the results of this state vector estimate for 738 the six parameters that are varied, transformed back to their biophysical meanings (through the 739 inverse of the functions in table 3). The sub-plots rows show results for the observation operator 740 parameters $\mathrm{LAI}, \mathrm{C}_{\mathrm{ab}}, \mathrm{C}_{\mathrm{w}} \mathrm{C}_{\mathrm{dm}}, \mathrm{N}$ and $\mathrm{s}_{1}$ respectively. The uncertainty (average credible interval) 741 associated with each (transformed) parameter for the baseline experiment is given in table 6 ('single 742 obs.'). Relating these uncertainties to the parameter ranges (table 4), we note that they are around 
$5 \%$ for $\mathrm{TLAI}, 10 \%$ for $\mathrm{s}_{1}$ and $\mathrm{TC}_{\mathrm{ab}}$ respectively for dates where there are observations, more than

$74420 \%$ for (transformed) leaf water and dry matter content and around $33 \%$ for N. We can suppose 745 these then to be typical uncertainty values for MSI sampling (with the assumed noise 746 characteristics). The cross correlation associated with these, illustrated in table 7 are highly variable 747 from one sample to the next. The median values given show quite strong negative correlations 748 between $\mathrm{TLAI}$ and $\mathrm{TC}_{\mathrm{ab}}$ and $\mathrm{TC}_{\mathrm{w}}$ but positive correlations with $\mathrm{s}_{1}$ and $\mathrm{TC}_{\mathrm{dm}}$. The median $\mathrm{s}_{1}$ shows 749 negative correlations with all terms other than TLAI. Despite the fact that the average transformed 750 LAI uncertainty is only around 5\%, we can see if figure 1 that both the error and uncertainty can be 751 rather high. Around peak LAI, results from individual samples vary by around LAI 2.5 and there is 752 a general tendency to underestimate. The general trends of $\mathrm{C}_{\mathrm{ab}}$ and $\mathrm{C}_{\mathrm{w}}$ are discernable, but there is 753 large variation and large uncertainty. The terms that are supposed to be constant here, $\mathrm{C}_{\mathrm{dm}}$ and $\mathrm{N}$ 754 depart significantly from their true state and the negative correlation is evident in the state 755 trajectories around the central part of the year.

TABLE 6 ABOUT HERE

TABLE 7 ABOUT HERE

How then can we improve on this situation? The ways to reduce uncertainty are to have data with lower noise characteristics, to average or smooth in some way, or to add other constraints to the solution. In any realistic scenario, we have only limited control of the first of these. Averaging and smoothing then are the general pragmatic responses to such issues. If however this is performed $a d$ $h o c$ as a post processing step to any individual term (e.g. only LAI) this would not take account of the cross correlation in the uncertainties which can only give sub-optimal results.

767 In spite of these quite high levels of uncertainty (and correlation of uncertainty) for these estimates, there is clearly quite a strong correlation between the values of the state vector and its neighbours in 
time. The general underlying patterns are apparent in the 'complete' scenario, although much of the

770 (potentially important) detail will be lost in a more realistic 'cloudy' scenario. The enhancement of

771 this temporal correlation effect and the suppression of the noise are at the heart of all regularisation approaches and the essence of weak constraint data assimilation. If we have some model

773 ('expectation') of the temporal trajectory of the state vector, then we can use this to filter the unwanted noise. As noted above, this may be a model based on our understanding of radiation interception and biogeochemical cycling (e.g. Quaife et al., 2008) driven by some set of external (environmental) parameters, or it may simply be some parametric curve that we believe can mimic e.g. the phenological development of LAI. In either case, what DA aims to achieve is an optimal merging of such models (through the adjustment of the state vector or essentially a calibration of the parameters controlling the development of state in the model) and the observations. For land surface monitoring, there are several options for such models for LAI development as mentioned, and up to a point for some other state variables (e.g. soil moisture), but there is very little to guide information extraction on many other state variables that affect the observations (e.g. leaf chlorophyll concentration or dry matter). In such a case, we need to develop simple methods, within a DA framework. Fortunately, there are many to choose from, although as Twomey (2002) points out, the results are likely to be similar for most of these methods: indeed, it would be worrying if they were not.

\subsection{DA: Complete scenario}

Here, we apply first order and second order derivative constraints to the solution, but we expect the results to be broadly similar. In both cases, we need only supply some estimate of the uncertainty associated with these constraints through the smoothness term to achieve a regularised solution to the state vector estimate. These constraints are applied by incorporating a model that, in the absence of any observations, would set the first (second) derivatives of the state variables to zero. Assuming that we apply the same (strength of) constraint to the whole time series, we need to supply an 
estimate of the mean squared first (second) difference in the parameter values (true values in table

796 5). For the first (second) difference then, this can be thought of as an estimate of the uncertainty in a 797 zero-order (first-order) process model over one time step as noted above.

We use a form of cross-validation to estimate . This is achieved with a synthetic dataset from an 800 alternative SPOT-5 HRG-like sensor. The core of the exercise then is a comparison between these 801 synthetic data (driven by the 'true' values of the state vector, plus random noise as above) and a simulation of the same sensor wavebands and acquisition geometry driven by the state vector estimated from the synthetic data from Sentinel-2 MSI. We choose this cross validation sensor as one different to MSI to stress that one role of a DA system of this sort can be to provide simulated data of sensors other than those used in the DA exercise. Here, we measure the average squared difference between the synthetic HRG data and the DA simulated observations, weighted by the uncertainty in the synthetic data, and term this RMSE in cross-validation. The locations of the synthetic HRG observations are indicated in the lower panel of figure 1 by + symbols.

FIGURE 2 ABOUT HERE

Figure 2 shows the error in cross-validation as a function of for the model first- and second-order

813 difference constraints for the complete case (black circles and squares respectively). There are clear

814 minima for these functions, which provide estimates of the optimal model uncertainty (averaged over all terms). Also shown in the figure is a set of vertical lines that represent the theoretical value of the smoothness term for each of the state vector elements that vary over time (from table 5). For

817 the first order constraint, the minimum of the cross validation function is $=150$ which is very close 818 to the theoretical values. For the second order constraint the cross validation RMSE minimum at $819=530$ is rather less than the theoretical values. For both cases however, we observe a very broad 
the synthetic cross validation HRG observations.

TABLE 8 ABOUT HERE

Tables 8 provides statistics on the uncertainty reduction, (the posterior uncertainty estimate from the DA relative to that after solving for each sample separately and assuming the prior uncertainty where there are no observations). The average improvement in uncertainty is 4.07 for the first order constraint and 2.73 for the second order difference constraint. This is very significant but it must be remembered that $4 / 5$ of the samples in the 'single obs' solution have only the prior constraint and uncertainty. Examining only locations where observation lie (i.e. ignoring interpolation

831 performance relative to the a priori estimate), we see the uncertainty reduction drop by nearly $50 \%$ 832 in this case, down to 2.20 for the first order constraint and 1.30 for the second difference constraint.

833 From those figures, we would suppose the first order constraint to be greatly superior to the second 834 order constraint, but if we look at the plots in figure 1, the second order constraint results seem to 835 have more reasonable uncertainty bounds than the other results. This is at least partially because the 836 apparent uncertainty resulting from the DA is strongly dependent on the value of used in the 837 model constraint: the higher the value of , the smoother will be the solution and the lower the 838 estimate of uncertainty. The only check we have done on the veracity of the solution comes from 839 the cross validation, which is an indirect check: in any non-synthetic experiment we rarely know the 840 'truth' to any great degree of certainty. Since we have a synthetic experiment here, we can however 841 test how frequently the derived solution matches the (synthetic) truth within the claimed uncertainty 842 bounds. One reasonable summary measure of this is the percentage of true values of state vector 843 elements that lie within the $95 \%$ credible interval claimed by the DA results. These are shown in 844 table 8 . We can see that for the 'single obs' estimates (no regularisation), only around $64 \%$ of the 845 state vector lies within the $95 \%$ credible interval claimed by the solution. The figure is as low as $84658 \%$ for $\mathrm{TC}_{\mathrm{dm}}$. We can suppose the average estimated uncertainty then to be only around $67 \%$ of 
847 the true value, i.e. we should inflate the estimated uncertainty by a factor of around 1.5 . This would

848 apply equally to the results in table 6 . We see almost the same value for the first order constraint,

849 which suggests the reduction in uncertainty by a factor of 2.2 is likely true. For the second order 850 difference constraint however, around 84\% (table 8) of the sample lie within the uncertainty bounds, 851 so here, a better estimate of the uncertainty reduction might be around 1.70 rather than the 1.30 852 reported. This apparent under-reporting of the uncertainty is worthy of comment and there could be 853 several reasons for this. One explanation could be that we are simply under-estimating the 854 uncertainty from the approximations made when calculating the Hessian for the observation 855 operator. A more likely reason is non-linear effects in the treatment of uncertainties. In spite of our 856 attempt to account for gross non-linear impacts through parameter transformations, residual non857 linear effects may be causing this under-estimation of uncertainty by a factor of around 1.5.

FIGURE 3 ABOUT HERE

FIGURE 4 ABOUT HERE

\subsection{DA: Cloudy scenario}

863 Figure 3 shows the DA results for the cloudy scenario. This is a much more realistic test for a DA 864 system. The task now is not only to reduce the uncertainty at the points where we have observations but also to try to provide an effective interpolation over data gaps. The cross validation plots for this case are shown in figure 2 (white circle and square) and provide a much more narrow minimum.

867 This implies that to achieve acceptable results in cross validation, the range of values that can be 868 tolerated is much more restricted. The minima of these functions however are well within the 869 bounds of the cross validation results for the 'complete' scenario and the optimal indicated very 870 similar to that obtained from the previous results. This indicates that the method for estimating is 871 quite robust, even when there are large data gaps. Unsurprisingly, the absolute value of the cross 872 validation RMSE is higher for the cloudy case, indicating poorer performance in prediction for this 
lower quality dataset.

\section{4}

875

876

877

878

879

880

881

882

883

884

885

886

887

888

889

890

891

892

893

894

895

896

897

898

\section{TABLE 9 ABOUT HERE}

Table 9 shows the reduction in uncertainty for this experiment. One striking feature of these is that the percentage of cases within the credible interval is now above $80 \%$ in both cases, meaning that the reported uncertainties are close to the true values. Whilst the apparent reduction in uncertainty is apparently quite small (indeed, there is an increase in uncertainty for some state vector elements) at 1.53 for the first order constraint and 1.14 for the second order, when weighed against the improved statistical representation, these rise to values directly comparable with the results from the previous experiment. The credible intervals shown in figure 3 are now realistic representations of the state vector elements and their uncertainties, achieved with only $50 \%$ of the samples of the previous experiment and with large data gaps, which is an important result.

Figure 4 shows the posterior correlation matrices (the inverse Hessian matrix) for the cloudy scenario. The general pattern of this matrix for the 'complete' scenario is rather similar so not shown here. Obviously, the correlation is unity along the leading diagonal. Another important feature is that the broad patterns of positive and negative correlations that we noted for the 'single obs' solutions remains here. There is negative correlation between $s_{1}$ and all terms by TLAI. There is negative correlation between TLAI and $\mathrm{TC}_{\mathrm{ab}}$ and $\mathrm{TC}_{\mathrm{w}}$ but positive correlation with $\mathrm{TC}_{\mathrm{dm}}$. These patterns are consistent for both constraints used. We notice then that the application of the dynamic model (regularisation) in time does not remove the correlations arising from the inverse Hessian of the observation cost function, but rather it 'spreads' uncertainty correlation out in the time domain. This is particularly visible in the second order constraint matrix in figure 4 where we can clearly see this smoothing being greater where there are data gaps ( $s_{1}$ is a good example of that). Equally, where a part of the state vector has been strongly influenced by the regularisation (e.g. $\mathrm{N}$ for the 
first order constraint) we see very high correlation at all time steps. Another interesting feature of

900 this figure is the fact that for some state vector elements (e.g. $\mathrm{N}$ for the second order constraint) we 901 can clearly see the influence of the periodic boundary condition).

\section{Discussion}

\subsection{The value of an EO-LDAS}

This paper outlines a scheme for a weak constraint data assimilation system, developed in the ESA

EO-LDAS project, designed for integrating Earth Observation data from a variety of sources over arbitrary time scales, and through that to multiple spatial resolutions. It has the potential, via careful definition of the underlying model to be extended to spatial constraints, although this is not explored here. The scheme is designed to allow interface with process models, should they be available, though only an empirical regularisation model is shown in this paper. The core of the system is a set of constraints on: (i) prior estimates of the state vector; (ii) a linear model of the state vector; (iii) observation operator (RT model) predictions of a set of EO data and a DA scheme

In this paper, we have set up and run a synthetic data experiment with EO data mimicking those that might be provided by the MSI sensors on the forthcoming Sentinel-2 platforms. Experiments in DA are conducted for an idealised 'full coverage' scenario (5 day sampling) and for a 'cloudy' case

918 (average around 10 day sampling but with large data gaps of up to 60 days). The results are 919 compared to baseline experiments where we attempt to estimate the state variable trajectories over 920 the course of a year for a subset of the total state variables (six elements per observation period). 921 The prior term is used only very weakly here, although bounds are set to the state vector elements. 922 Further, we assume that we have direct access to the surface reflectance (as opposed to top of 923 atmosphere radiance), and that the noise on the observations is uncorrelated and of known 924 magnitude. Broadly however, we can claim that the baseline results should be indicative of those 
that might be obtained from Sentinel-2 data using 'traditional' estimation methods. For what we suppose to be a typical observation noise scenario, the uncertainty can be a quite large proportion of

927 the signal for important terms such as LAI, this for a peak LAI of only around 3.7, although on 928 average the uncertainty in TLAI may only be around 5\%. This then, relates to the information content of a single MSI observation for this level of noise, assuming some important terms such as leaf angle distribution are known precisely. These results are not surprising but are simply a manifestation of the difficulty of the inference of biophysical parameters from remote radiometric observations: the problem may often be ill-posed (consider the situation if only two wavebands at red and near infrared were available), but even if it is not strictly so, there may not be sufficient information to very well constrain the information we require. In any case, there can be quite high correlation in uncertainty.

The ways to improve this situation are: (i) to obtain more observations (although more observations does not always translate to more information: consider again sampling at only red and near infrared wavelengths in trying to constrain e.g. leaf water content); or (ii) to add some other forms of information; (iii) average the data. Much a priori information has been used in the past to help

941 constrain these problems, but this has often been approached in a rather ad hoc manner. Examples

942 include: assuming some terms known, without considering the impact of uncertainty in these, or 943 imposing degrees of smoothness; assuming that some terms are constant over some arbitrary time 944 period; or post hoc low pass filtering to the final results. Given its success in other field of science 945 and engineering, many authors have proposed that DA should be seen as the route to integration of 946 the various forms of information one might wish to use to constrain the estimation. Key to DA is the 947 weighting between the various sources of evidence, and key to this is assigning uncertainty 948 correctly to the sources. This is a feature of the approach that dramatically differentiates it from the 949 way in which VIs are mostly used in EO. As we note in the introduction, if we wish to estimate biophysical parameters (such as LAI) there is generally some form of calibration (against ground 
observations or RT model runs) but it is extremely rare that those model uncertainties are

952 considered in mapping the product. Other processing steps such as angular normalisation may have taken place, but again, any concepts of uncertainty arising from these are on the whole disregarded.

954 All of these issues could be addressed within a DA framework, even if the source of the EO information were to be VIs.

957 If a biophysical process model is available to predict the development of the state variables that 958 control the remote sensing signal, this can clearly add information to help constrain the problem. If 959 information from the observations feed back to improve the estimates of the parameters controlling 960 the process model or alternatively improve the state estimates, then a better integration of 961 observations and model is achieved, which will likely better constrain additional terms estimated by 962 the process model. This has been argued by Quaife et al. (2008) and others who have worked on 963 integrating EO data and e.g. Carbon flux process models. However, models such as these simply do 964 not provide information on a large number of the variables that affect EO signals, and this is likely 965 to remain the case for the foreseeable future. Exercises in EO-model integration then 966 understandably tend to focus of the points of common linkage (which often is no more than LAI, 967 being supposed linearly related to leaf Carbon) and then applying the 'traditional' methods to the 968 remaining parameters (assuming them known or at best constant over time). In this paper, and in the 969 EO-LDAS work in general, we have taken the focus away from working with some specific process 970 model, and tried to consider the more general case and the sorts of constraints that might be 971 appropriate. If no physical model is available, empirical concepts of smoothness in the state 972 variables come to the fore. These ideas become even more important if one considers constraint in 973 the spatial domain, where physical or even biological process models are almost completely lacking 974 to aid biophysical parameter estimation.

976 The EO-LDAS scheme that we have built is capable of using any linearised process model and of 
more general interface to process model codes provided the cost function and its derivatives can be

978 calculated. In the prototype and in this paper we have examined first- and second-order derivative

979 constraints as general, appropriate (empirical) models for biophysical parameter estimation in DA.

980 We have simulated typical profiles of LAI and leaf chlorophyll concentration and rather complex

981 profiles of leaf water concentration and soil brightness and shown that with Sentinel-2 MSI data

982 every 5 days, a reduction in uncertainty by a factor of around 2 might generally be achieved. More

983 interestingly perhaps, after compensation for errors in uncertainty prediction, we saw that similar

984 reductions might be achieved even when there are large data gaps and 50\% of the samples lost due

985 to cloud cover.

987 We have also demonstrated (figure 2) that it is feasible to estimate the required hyper-parameters

988 from some form of cross-validation exercise to impose an appropriate degree of model uncertainty, 989 and that quite consistent results can be obtained even under cloudy conditions. This is an important 990 practical point for the eventual operationalisation of these methods, but the area requires a little 991 more discussion of practical issues in its implementation.

993 Approximate linearization of the RT model variables here, following Weiss et al. (2000), has 994 allowed Gaussian distributions to be assumed throughout. Although we have not directly 995 investigated any residual non-linear effects in this study, some evidence is provided that on average 996 we may be predicting only around $2 / 3$ of the true uncertainty.

\section{$998 \quad 4.1$ Future directions}

999 In this paper, we have only demonstrated DA for a homogeneous observation system, i.e. one for 1000 which we have assumed the spectral sampling (and in effect, spatial resolution) for all observations 1001 is the same. Using the EO-LDAS prototype for spectrally heterogeneous systems is straightforward, 1002 but further work is needed to test the multi-scale concepts that would more generally be required. 
1003 Within the existing prototype, the state vector can represent any mixture of temporal or spatial 1004 samples. The concepts of temporal smoothness used here apply equally to the spatial domain 1005 (indeed, such ideas form the basis of the field of geostatistics (e.g. Atkinson and Lewis, 2000)), so 1006 the prototype can be used directly to link a state vector representation on a spatial grid, via 1007 appropriate specification of the matrix $A$. Indeed, one could consider the experiments performed in 1008 this paper simply as being on a spatial transect, rather than as we have assumed a temporal sampling 1009 pattern. The only practical difference is that in that case, the viewing and illumination angles would 1010 be near identical for all samples.

1012 The EO-LDAS prototype is designed to allow a (relatively) large number of state variables to be 1013 estimated simultaneously in a variational system (> 2000 demonstrated here). One potential 1014 advantage of this is that information can be passed between any of the state vector elements. In 1015 practice, we have only used rather local information transfer in the model constraints applied here 1016 (differences with neighbours in time) and this approach could also be implemented as a sequential 1017 smoother. In viewing the temporal experiment we have performed as effectively equivalent to a 1018 spatial experiment, the neighbourhood need not be very different (i.e., in the spatial sense, we could 1019 follow the approach here and directly connect information in one grid cell to its 8 neighbours). 1020 However, this variational system maintains the capacity for more distant (time or space) 1021 connections, for example in applying multiple scale constraint.

1023 A point that we have not dwelt on in this paper is the time required for processing. This is currently 1024 around several hours for solving for $>2000$ state vector elements using 73 samples for what equates 1025 to a single pixel (albeit for all samples over a year). The experiments in this paper were conducted 1026 over around 120 UNIX cores, so quite large-scale experiments are feasible using University 1027 computing resources. Clearly the processing requirements would need to be greatly reduced if such 1028 a system were to be proposed for operational processing. The computer code is not on the whole 
1029 written to be fast, but rather to be adequate to learn about using this form of DA. There are various 1030 ways in which this might be tackled: clearly the very tight convergence criteria could be somewhat 1031 relaxed, and more efficient codes could be written, but there will always be a relatively large 1032 overhead on multiple calculations of a radiative transfer model. Pragmatic ways to overcome this 1033 issue have mainly in the past dealt with using LUTs or ANNs to sample or approximate the 1034 observation operator, but clearly in the DA framework we must consider representational error in 1035 any such emulation. One avenue that holds much promise is that of Gaussian Process (GP) 1036 emulators (Kennedy and O'Hagan, 2000, 2001), a form of regression that has been successfully 1037 used to simulate computationally costly models runs through simple functional approximations. The 1038 great benefit of this latter approach is that uncertainties in the emulated model are included and that 1039 derivatives of the model can also be easily produced. If we consider the observation operator as a 1040 sampled function with GP emulation, it is interesting to note that the underlying concepts implying 1041 smooth interpolation with treatment of representation uncertainty are of course the same as we are 1042 performing in the temporal (or indeed spatial) process model in the DA.

\section{Conclusions}

1045 The EO-LDAS prototype that is described in this paper has been demonstrated to be capable of 1046 simultaneously estimating a state vector of over 2000 elements of surface biophysical 1047 characteristics in a synthetic experiment using simulated Sentinel-2 MSI data. Although the 1048 processing time required for this is currently a little long, this is a significant step in the size of such 1049 problems that can be tackled simultaneously. The ability to do this derives from the use of AD1050 generated adjoint code for the observation operator at the heart of the DA system.

1052 The DA scheme that has been developed is a weak constraint variational system. The value of such 1053 a scheme has been demonstrated using the synthetic MSI data to show a reduction in uncertainty of 1054 up to around 2 when a linear dynamic model is used in the DA. The linear dynamic model is 
1055

1056

1057

1058

1059

1060

1061

1062

1063

1064

1065

1066

1067

1068

1069

1070

1071

1072

1073

1074 There is clearly quite a long way to go from initial experiments with relatively slow computer codes 1075 1076 1077 1078

proposed as a general implementation that can potentially be interfaced to biophysical process models through linearization. It is used in this paper with first and second-order derivative constraints (zero- and first-order process models) which are shown to be sufficient to track rather complex biophysical parameter trajectories via a radiative transfer model 'observation operator' interface to the synthetic EO data.

We have noted at various points in this text, that some aspects of the EO-LDAS prototype are still under development of testing, but what actually is provided by the prototype code is a functioning tool for exploring many issues in DA and for estimating information on surface biophysical parameters. The tool is designed as a weak constraint variational system, but we have argued that it can also be used sequentially as it stands. We have demonstrated the use of the tool and of DA concepts in reducing uncertainty in biophysical parameter estimation in a temporal sense, but also argued the equivalence of this (in DA in general, but in the tool specifically) for the spatial domain as well. We have used only empirical 'regularisation' concepts in demonstrating the DA, but noted that these are powerful general concepts that are extremely useful, particularly if biophysical models do not treat some of the parameters we are concerned with. In the more general case though, any linearization of a more process-driven model can be directly interfaced to the EO-LDAS prototype.

to an operational system for land data information extraction from EO, i.e. an operational EOLDAS, but the concepts explored here demonstrate the power and potential flexibility of such an approach.

\section{Acknowledgements}

1080 We gratefully acknowledge the support of ESA through the EO-LDAS project 22205/09/I-EC for 
1081 funding this work. We also acknowledge the support of the (UK) National Environment Research

1082 Council (NERC) National Centre for Earth Observation (NCEO) for its support of several of the 1083 personnel involved in this work. We would further like to thank the attendees of the EO-LDAS 1084 Community Workshop held in ESA ESRIN in November 2009 for their feedback and inputs to this 1085 study.

1087 References

1088 Asrar, G., Kanemasu, E.T., Jackson, R.D., Pinter Jr, P.J., 1985. Estimation of total above-ground 1089 phytomass production using remotely sensed data. Remote Sensing of Environment 17, 211-220.

1090 Atkinson, P. and Lewis, P., 2000, Geostatistical classification for remote sensing: an introduction. 1091 Computers and Geoscience, 26(4), 361-371.

1092 Baret, F. and Guyot, G., 1991. Potentials and limits of vegetation indices for LAI and APAR 1093 assessment. Remote Sensing of Environment 35, 161-173.

1094 Behrenfeld, M.J., Randerson, J.T., McClain, C.R., Feldman, G.C., Los, S.O., Tucker, C.J., 1095 Falkowski, P.G., Field, C.B., Frouin, R., Esaias, W.E., others, 2001. Biospheric primary production 1096 during an ENSO transition. Science 291, 2594.

1097 Beven, K., 2006. A manifesto for the equifinality thesis. Journal of Hydrology 320, 18-36.

1098 Byrd, R.H., Lu, P., Nocedal, J., Zhu, C., 1995. A limited memory algorithm for bound constrained 1099 optimization. SIAM Journal on Scientific Computing 16, 1190-1208.

1100 CEOS, 2011a. The CEOS Constellation for Land Surface Imaging [WWW Document]. URL 1101 http://wgiss.ceos.org/lsip/lsic.shtml, accessed 18/11/2011.

1102 CEOS, 2011b, CEOS EO Handbook - Instrument summary: HRG. [WWW Document]. URL 1103 http://database.eohandbook.com/database/instrumentsummary.aspx?instrumentID=183 accessed 1104 18/11/2011

1105 Chen, J.M., Pavlic, G., Brown, L., Cihlar, J., Leblanc, S.G., White, H.P., Hall, R.J., Peddle, D.R., 1106 King, D.J., Trofymow, J.A., others, 2002. Derivation and validation of Canada-wide coarse- 
1107 resolution leaf area index maps using high-resolution satellite imagery and ground measurements.

1108 Remote Sensing of Environment 80, 165-184.

1109 Choudhury, B.J., 1987. Relationships between vegetation indices, radiation absorption, and net 1110 photosynthesis evaluated by a sensitivity analysis. Remote Sensing of Environment 22, 209-233.

1111 Clerici, M., Vossbeck, M., Pinty, B., Kaminski, T., Taberner, M., Lavergne, T., Andredakis, I., 2010. 1112 Consolidating the two-stream inversion package (JRC-TIP) to retrieve land surface parameters from 1113 albedo products. Selected Topics in Applied Earth Observations and Remote Sensing, IEEE Journal 1114 of 3, 286-295.

1115 Combal, B., Baret, F., Weiss, M., Trubuil, A., Mace, D., Pragnere, A., Myneni, R., Knyazikhin, Y., 1116 Wang, L., 2003. Retrieval of canopy biophysical variables from bidirectional reflectance:: Using 1117 prior information to solve the ill-posed inverse problem. Remote Sensing of Environment 84, 1-15. 1118 Council of the European Union, 2010. Taking forward the European Space Policy.

1119 DMCII, 2010. DMC Constellation [WWW Document]. URL 1120 http://www.dmcii.com/about_us_constellation.htm, accessed 20/12/2010.

1121 Eilers, P.H.C., 2003. A perfect smoother, Analytical Chemistry, 75(14), pp. 3631-3636

1122 Enting, I.G., 2002. Inverse problems in atmospheric constituent transport. Cambridge University 1123 Press.

1124 ESA, 2010. Mission Requirements Document GMES Sentinel-2 [WWW Document]. URL 1125 http://esamultimedia.esa.int/docs/GMES/Sentinel-2_MRD.pdf, accessed 20/12/2010.

1126 European Comission, n.d. GEOSS: Policy relevance, Future Challenges, EU contribution to 1127 GEOSS, Relevant documentation [WWW Document]. GEOSS. URL 1128 http://ec.europa.eu/research/environment/index_en.cfm?section=geo\&pg=geoss, accessed $112920 / 12 / 2010$.

1130 Evensen, G., 2003. The ensemble Kalman filter: Theoretical formulation and practical 1131 implementation. Ocean dynamics 53, 343-367.

1132 Fang, H., Liang, S., Hoogenboom, G., Teasdale, J., Cavigelli, M., 2008a. Corn-yield estimation 
1133 through assimilation of remotely sensed data into the CSM-CERES-Maize model. International 1134 Journal of Remote Sensing 29, 3011.

1135 Fang, H., Liang, S., Townshend, J.R., Dickinson, R.E., 2008b. Spatially and temporally continuous 1136 LAI data sets based on an integrated filtering method: Examples from North America. Remote 1137 Sensing of Environment 112, 75-93.

1138 Féret, J.B., François, C., Asner, G.P., Gitelson, A.A., Martin, R.E., Bidel, L.P., Ustin, S.L., le Maire, 1139 G., Jacquemoud, S., 2008. PROSPECT-4 and 5: Advances in the leaf optical properties model 1140 separating photosynthetic pigments. Remote Sensing of Environment 112, 3030-3043.

1141 Fung, A.K. and Chen, K., 2010. Microwave Scattering and Emission Models for Users, 1 st ed. 1142 Artech House Publishers.

1143 Garrigues, S., Lacaze, R., Baret, F., Morisette, J.T., Weiss, M., Nickeson, J.E., Fernandes, R., 1144 Plummer, S., Shabanov, N.V., Myneni, R.B., others, 2008. Validation and intercomparison of global 1145 Leaf Area Index products derived from remote sensing data. Journal of Geophysical Research, 113, 1146 G02028.

1147 Ghil, M. and Malanotte-Rizzoli, P., 1991. Data assimilation in meteorology and oceanography. Adv. 1148 Geophys 33, 141-266.

1149 Giering, R. and Kaminski, T., 1998. Recipes for adjoint code construction. ACM Transactions on 1150 Mathematical Software (TOMS) 24, 437-474.

1151 Gill, P.E., Murray, W., Wright, M.H., 1981. Practical optimization. Academic Press, London and 1152 New York.

1153 Gobron, N., Pinty, B., Verstraete, M.M., Govaerts, Y., 1997. A semidiscrete model for the scattering 1154 of light by vegetation. Journal of Geophysical Research 102, 9431-9446.

1155 Gobron, N., Pinty, B., Verstraete, M., Widlowski, J., 2000. Advanced vegetation indices optimized 1156 for up-coming sensors: Design, performance, and applications. IEEE Transactions on Geoscience 1157 and Remote Sensing, 38, 2489-2505.

1158 Gobron, N., Pinty, B., Verstraete, M.M., Widlowski, J.L., 2002. Advanced vegetation indices 
1159 optimized for up-coming sensors: Design, performance, and applications. IEEE Transactions on 1160 Geoscience and Remote Sensing, 38, 2489-2505.

1161 Gobron, N., Belward, A., Pinty, B., Knorr, W., 2010. Monitoring biosphere vegetation 1998-2009. 1162 Geophysical Research Letters 37, L15402.

1163 Goel, N.S., 1988. Models of vegetation canopy reflectance and their use in estimation of 1164 biophysical parameters from reflectance data. Remote Sensing Reviews 4, 1-212.

1165 Goel, N.S., Thompson, R.L., 2000. A snapshot of canopy reflectance models and a universal model 1166 for the radiation regime. Remote Sensing Reviews 18, 197-225.

1167 Goward, S.N., Tucker, C.J., Dye, D.G., 1985. North American vegetation patterns observed with the 1168 NOAA-7 advanced very high resolution radiometer. Plant Ecology 64, 3-14.

1169 Jacquemoud, S., Baret, F., 1990. PROSPECT: A model of leaf optical properties spectra. Remote 1170 Sensing of Environment 34, 75-91.

1171 Kennedy, M.C., O'Hagan, A., 2001. Bayesian calibration of computer models. Journal of the Royal 1172 Statistical Society: Series B (Statistical Methodology) 63, 425-464.

1173 Kennedy, M.C., O'Hagan, A., 2000. Predicting the output from a complex computer code when fast 1174 approximations are available. Biometrika 87, 1.

1175 Kimes, D.S., Knyazikhin, Y., Privette, J.L., Abuelgasim, A.A., Gao, F., 2000. Inversion methods for 1176 physically-based models. Remote Sensing Reviews 18, 381-439.

1177 Knorr, W., Kaminski, T., Scholze, M., Gobron, N., Pinty, B., Giering, R., Mathieu, P., 2010. Carbon 1178 cycle data assimilation with a generic phenology model. Journal of Geophysical. Research, 115, 1179 G04017.

1180 Kuusk, A., 1995. A fast, invertible canopy reflectance model. Remote Sensing of Environment 51, $1181342-350$.

1182 Lavergne, T., Kaminski, T., Pinty, B., Taberner, M., Gobron, N., Verstraete, M.M., Vossbeck, M., 1183 Widlowski, J.L., Giering, R., 2007. Application to MISR land products of an RPV model inversion 1184 package using adjoint and Hessian codes. Remote Sensing of Environment 107, 362-375. 
1185 Lewis, P. and Disney, M., 2007. Spectral invariants and scattering across multiple scales from 1186 within-leaf to canopy. Remote Sensing of Environment 109, 196-206.

1187 Liang, S. and Strahler, A.H., 2002. An analytic BRDF model of canopy radiative transfer and its 1188 inversion. IEEE Transactions on Geoscience and Remote Sensing, 31, 1081-1092.

1189 Lu, X., Liu, R., Liu, J., Liang, S., 2007. Removal of noise by wavelet method to generate high 1190 quality temporal data of terrestrial MODIS products. Photogrammetric Engineering and Remote 1191 Sensing 73, 1129.

1192 Lubansky, A. S., Yeow, Y. L., Leong Y-K., Wickramasinghe S. R., Han B., 2006. A general method 1193 of computing the derivative of experimental data., AIChe J., 52, pp. 323-332.

1194 Lyapustin, A. and Knyazikhin, Y., 2001, Method of Green Function in the Radiative Transfer 1195 Problem. Part I: Homogeneous non-Lambertian Surface. Applied Optics, 40, 3495-3501.

1196 Lyapustin, A., Wang, Y., Martonchik, J., Privette, J., Holben, B., Slutsker, I., Sinyuk, A. and 1197 Smirnov, A., 2006, Local analysis of MISR surface BRF and albedo over GSFC and Mongu 1198 AERONET sites, IEEE Transactions on Geoscience and Remote Sensing, 44, 1707-1718.

1199 McLaughlin, D., 2002. An integrated approach to hydrologic data assimilation: interpolation, 1200 smoothing, and filtering. Advances in Water Resources 25, 1275-1286.

1201 Myneni, R.B., Maggion, S., Iaquinta, J., Privette, J.L., Gobron, N., Pinty, B., Kimes, D.S., 1202 Verstraete, M.M., Williams, D.L., 1995. Optical remote sensing of vegetation: Modeling, caveats, 1203 and algorithms. Remote Sensing of Environment 51, 169-188.

1204 NASA, 2010. NASA - A-Train [WWW Document]. URL 1205 http://www.nasa.gov/mission_pages/cloudsat/multimedia/a-train.html

1206 Nemani, R.R., Keeling, C.D., Hashimoto, H., Jolly, W.M., Piper, S.C., Tucker, C.J., Myneni, R.B., 1207 Running, S.W., 2003. Climate-driven increases in global terrestrial net primary production from 12081982 to 1999 . Science $300,1560$.

1209 Olioso, A., Inoue, Y., Ortega-Farias, S., Demarty, J., Wigneron, J.P., Braud, I., Jacob, F., 1210 Lecharpentier, P., Ottlé, C., Calvet, J.C., others, 2005. Future directions for advanced 
1211 evapotranspiration modeling: Assimilation of remote sensing data into crop simulation models and 1212 SVAT models. Irrigation and Drainage Systems 19, 377-412.

1213 Pinty, B., Lavergne, T., Vossbeck, M., Kaminski, T., Aussedat, O., Giering, R., Gobron, N., 1214 Taberner, M., Verstraete, M.M. and Widlowski, J-L. (2007) Retrieving surface parameters for 1215 climate models from Moderate Resolution Imaging Spectroradiometer (MODIS)-Multiangle 1216 Imaging Spectroradiometer (MISR) albedo products, Journal of Geophysical. Research, 112, 1217 D10116, doi:10.1029/2006JD008105.

1218 Price, J.C., 1990. On the information content of soil reflectance spectra. Remote sensing of 1219 Environment 33, 113-121.

1220 Privette, J.L., Myneni, R.B., Tucker, C.J., Emery, W.J., 1994. Invertibility of a 1-D discrete 1221 ordinates canopy reflectance model. Remote Sensing of Environment 48, 89-105.

1222 Qin, J., Liang, S., Li, X., Wang, J., 2008. Development of the adjoint model of a canopy radiative 1223 transfer model for sensitivity study and inversion of leaf area index. IEEE Transactions on 1224 Geoscience and Remote Sensing, 46, 2028-2037.

1225 Qin, J., Liang, S., Liu, R., Zhang, H., Hu, B., 2007. A weak-constraint-based data assimilation 1226 scheme for estimating surface turbulent fluxes. IEEE Geoscience and Remote Sensing Letters, 4, $1227 \quad 649-653$.

1228 Qin, J., Liang, S., Yang, K., Kaihotsu, I., Liu, R., Koike, T., 2009. Simultaneous estimation of both 1229 soil moisture and model parameters using particle filtering method through the assimilation of 1230 microwave signal. Journal of Geophysical Research 114, D15103.

1231 Quaife, T., Lewis, P., 2010. Temporal Constraints on Linear BRDF Model Parameters. IEEE 1232 Transactions on Geoscience and Remote Sensing, 48, 2445-2450.

1233 Quaife, T., Lewis, P., De Kauwe, M., Williams, M., Law, B.E., Disney, M., Bowyer, P., 2008. 1234 Assimilating canopy reflectance data into an ecosystem model with an Ensemble Kalman Filter. 1235 Remote Sensing of Environment 112, 1347-1364.

1236 Richardson, A.J., Wiegand, C.L., 1977. Distinguishing vegetation from soil background 
1237 information(by gray mapping of Landsat MSS data). Photogrammetric Engineering and Remote 1238 Sensing 43, 1541-1552.

1239 Rochdi, N., Fernandes, R., 2010. Systematic mapping of Leaf Area Index across Canada using 2501240 meter MODIS data. Remote Sensing of Environment 114, 1130-1135.

1241 Rodgers, C.D., 2000. Inverse Methods for Atmospheric Sounding : Theory and Practice. World 1242 Scientific Publishing Company.

1243 Roy, D.P., Jin, Y., Lewis, P.E., Justice, C.O., 2005. Prototyping a global algorithm for systematic 1244 fire-affected area mapping using MODIS time series data. Remote Sensing of Environment 97, 1371245162.

1246 Slater, A.G. and Clark, M.P., 2009. Snow data assimilation via an ensemble Kalman filter. Journal of 1247 Hydrometeorology, 7, 478-492.

1248 Sobrino, J.A., Jiménez-Muñoz, J.C., Verhoef, W., 2005. Canopy directional emissivity: Comparison 1249 between models. Remote Sensing of Environment 99, 304-314.

1250 Stöckli, R., Rutishauser, T., Dragoni, D., O'Keefe, J., Thornton, P.E., Jolly, M., Lu, L., Denning, 1251 A.S., 2008. Remote sensing data assimilation for a prognostic phenology model. Journal of 1252 Geophysical Research, 113, 19 PP.

1253 Tarantola, A., 2005. Inverse problem theory and methods for model parameter estimation. Society 1254 for Industrial and Applied Mathematics.

1255 Tha Paw, U., others, 1992. Development of models for thermal infrared radiation above and within 1256 plant canopies. ISPRS Journal of Photogrammetry and Remote Sensing, 47, 189-203.

1257 Tucker, C.J., 1979. Red and photographic infrared linear combinations for monitoring vegetation. 1258 Remote sensing of Environment, 8, 127-150.

1259 Twomey, S., 2002. Introduction to the Mathematics of Inversion in Remote Sensing. Courier Dover 1260 Publications.

1261 Verhoef, W., Bach, H., 2003. Simulation of hyperspectral and directional radiance images using 1262 coupled biophysical and atmospheric radiative transfer models. Remote Sensing of Environment, 
$87,23-41$.

1264 Vermote, E.F., Tanré, D., Deuzé, J.L., Herman, M., Morcrette, J.J., 1997. Second Simulation of the 1265 Satellite Signal in the Solar Spectrum, 6S: An Overview. IEEE Transactions on Geoscience and 1266 Remote Sensing, 35(3), 675-686.

1267 Vermote, E.F., El Saleous, N.Z., Justice, C.O., 2002. Atmospheric correction of MODIS data in the 1268 visible to middle infrared: first results. Remote Sensing of Environment, 83, 97-111.

1269 Wahba, G., 1990. Spline models for observational data. Philadelphia, Pa: Society for Industrial and 1270 Applied Mathematics.

1271 Weiss, M., Baret, F., Myneni, R.B., Pragnère, A., Knyazikhin, Y., 2000. Investigation of a model 1272 inversion technique to estimate canopy biophysical variables from spectral and directional 1273 reflectance data, Agronomie 20, 3-22.

1274 Widlowski, J.L., Taberner, M., Pinty, B., Bruniquel-Pinel, V., Disney, M., Fernandes, R., Gastellu1275 Etchegorry, J.P., Gobron, N., Kuusk, A., Lavergne, T., others, 2007. Third Radiation Transfer Model 1276 Intercomparison (RAMI) exercise: Documenting progress in canopy reflectance models. Journal of 1277 Geophysical Research, 112, D09111.

1278 Xiao, Z., Liang, S., Wang, J., Song, J., Wu, X., 2009. A temporally integrated inversion method for 1279 estimating leaf area index from MODIS data. IEEE Transactions on Geoscience and Remote 1280 Sensing, 47, 2536-2545.

1281 Xiao, Z., Liang, S., Wang, J., Jiang, B., Li, X., 2011. Real-time retrieval of Leaf Area Index from 1282 MODIS time series data. Remote Sensing of Environment 115, 97-106.

1283 Zhu, C., Byrd, R.H., Lu, P., Nocedal, J., 1997. Algorithm 778: L-BFGS-B: Fortran subroutines for 1284 large-scale bound-constrained optimization. ACM Transactions on Mathematical Software (TOMS) $128523,550-560$.

1286 Zupanski, D.., 1997, A general weak constraint applicable to operational 4dvar data assimilation 1287 systems, Monthly weather review, 125, 2274-2292. 
1289

1290 
Table Captions

1292 Table 1. Spatial resolution, Central wavelength and bandwidths for Sentinel-2 MSI (ESA, 2010).

1293 Table 2. Summary of observation operator state variables.

1294 Table 3. Transformations applied to approximate linearise state variable response.

1295 Table 4. Upper and lower bounds for the state vector terms (in transformed space, where 1296 appropriate) used in the simulations, along with the temporal trajectory assumed.

1297 Table 5. Model uncertainty $\gamma$ for each parameter, calculated from the synthetic model state vector.

$1298 \mathrm{TC}_{\mathrm{dm}}$ and $\mathrm{N}$ were kept constant, so have no theoretical model uncertainty associated.

1299 Table 6: Mean posterior uncertainty. Figures refer to the complete daily time series, while figures in 1300 brackets refer to the mean posterior uncertainty only considering the dates where observations are 1301 available.

1302 Table 7: Single observation posterior correlation matrix. Elements above the main diagonal show 1303 the results for DoY 186, whereas the elements below the main diagonal represent the median of all 1304 dates.

1305 Table 8: Uncertainty reduction relative to the single observation inversion, as well as percentage of 1306 cases where the true parameter lies within the estimated 95\% credible interval. Results for non1307 cloudy scenario are reported. both complete time series.

1308 Table 9: Uncertainty reduction relative to the single observation, as well as percentage of cases 1309 where the true parameter lies within the estimated 95\% confidence interval. Results for cloudy 1310 scenario. 


\section{Figure Captions}

1319 Figure 1. Base level state vector estimated from inverting single observations, (left column) and for 1320 model uncertainty unknown and estimated through cross-validation - first difference constraint 1321 (central column) and second difference constraint (third column). Results for each of the six 1322 parameters are shown in rows. True values are shown as a dashed line. The full lines are the 1323 posterior means, and the shaded area represents the associated +/-1.96 standard deviations interval. 1324 MSI observations are shown as open symbols. Crosses along the bottom of the third row indicate 1325 the location of the cross validation acquisition dates.

1326 Figure 2. Error in cross validation scaled by observational uncertainty for varying model uncertainty $1327 \gamma$ for first and second order constraints. Vertical lines around 200 represent the theoretical value of $\gamma$ 1328 for each of the 4 time-varying state variables using a first order constraint, and vertical lines around 13295000 represent the theoretical values for $\gamma$ for each of the 4 time-varying state variables using 1330 second order constraint.

1331 Figure 3. Base level state vector estimated from inverting single observations, (left column) and for 1332 model uncertainty unknown and estimated through cross-validation - first difference constraint 1333 (central column) and second difference constraint (third column). Reduced number of acquisitions 1334 due to cloud cover scenario. Results for each of the six parameters are shown in rows. True values 1335 are shown as a dashed line. The full lines are the posterior means, and the shaded area represents the 1336 associated +/-1.96 standard deviations interval. MSI observations are shown as open symbols. 1337 Crosses along the bottom of the third row indicate the location of the cross validation acquisition 1338 dates.

1339 Figure 4. Posterior correlation matrices for the cloudy scenario. Labels indicate the location of the 1340 first day for component of the state vector. 
Table 1. Spatial resolution, Central wavelength and bandwidths for Sentinel-2 MSI (ESA, 2010).

\begin{tabular}{|l|l|l|l|l|l|l|l|l|l|l|l|l|l|}
\hline$\#$ & 1 & 2 & 3 & 4 & 5 & 6 & 7 & 8 & $8 \mathrm{a}$ & 9 & 10 & 11 & 12 \\
\hline $\begin{array}{l}\text { Spatial } \\
\text { Resolution }\end{array}$ & 60 & 10 & 10 & 10 & 20 & 20 & 20 & 10 & 20 & 60 & 60 & 20 & 20 \\
\hline $\begin{array}{l}\text { Wavelength } \\
\text { / nm }\end{array}$ & 443 & 490 & 560 & 665 & 705 & 740 & 783 & 842 & 865 & 945 & 1375 & 1610 & 2190 \\
\hline $\begin{array}{l}\text { Bandwidth } \\
\text { /nm }\end{array}$ & 20 & 65 & 35 & 30 & 15 & 15 & 20 & 115 & 20 & 20 & 30 & 90 & 180 \\
\hline
\end{tabular}

Table 2. Summary of observation operator state variables. 


\begin{tabular}{|c|c|c|c|c|c|c|}
\hline \# & Name & Symbol & Units & $\begin{array}{l}\text { Default } \\
\text { value }\end{array}$ & Lower limit & $\begin{array}{l}\text { Upper } \\
\text { limit }\end{array}$ \\
\hline 1 & $\begin{array}{ll}\text { Leaf } & \text { Area } \\
\text { Index } & \end{array}$ & LAI & none & 0.01 & 0.01 & 5.4 \\
\hline 2 & $\begin{array}{l}\text { Canopy } \\
\text { height }\end{array}$ & $x h$ & $\mathrm{~m}$ & 5 & 1.0 & 5 \\
\hline 3 & Leaf radius & $x r$ & $m$ & 0.01 & 0.001 & 0.1 \\
\hline 4 & $\begin{array}{l}\text { Chlorophyll } \\
a, b\end{array}$ & $\mathrm{C}_{\mathrm{ab}}$ & $\mu \mathrm{gcm}^{-2}$ & 40 & 0 & 200 \\
\hline 5 & Carotenoids & $\mathrm{C}_{\mathrm{ar}}$ & $\mu \mathrm{gcm}^{-2}$ & 0 & 0 & 200 \\
\hline 6 & Leaf water & $\mathrm{C}_{\mathrm{w}}$ & $\mathrm{cm}^{-1}$ & 0.01 & 0.00001 & 0.04 \\
\hline 7 & Dry matter & $C_{m}$ & $\mathrm{gcm}^{-2}$ & 0.01 & 0.00001 & 0.02 \\
\hline 8 & Leaf layers & $N$ & none & 1.0 & 1.0 & 2.5 \\
\hline 9 & soil PC 1 & $s_{1}$ & none & 0.2 & 0.05 & 0.4 \\
\hline 10 & soil PC 2 & $s_{1}$ & none & 0 & -0.1 & 0.1 \\
\hline 11 & soil PC 3 & $s_{1}$ & none & 0 & -0.05 & 0.05 \\
\hline 12 & soil PC 4 & $s_{1}$ & none & 0 & -0.03 & 0.03 \\
\hline 13 & $\begin{array}{l}\text { Leaf angle } \\
\text { distribution } \\
\text { (categorised) }\end{array}$ & $\mathrm{g}$ & none & uniform & $\begin{array}{l}\text { 1. planophile } \\
\text { 2. erectophile } \\
\text { 3. plagiophile } \\
\text { 4. extremophile } \\
\text { 5. uniform }\end{array}$ & $\mathrm{n} / \mathrm{a}$ \\
\hline
\end{tabular}


Table 3. Transformations applied to approximate linearise state variable response

\begin{tabular}{|l|l|l|}
\hline$\#$ & Transformed & Transformation \\
\hline $\mathbf{1}$ & TLAI & $\exp (-\mathrm{LAI} / 2.0)$ \\
\hline $\mathbf{4}$ & $\mathrm{TC}_{\mathrm{ab}}$ & $\exp \left(-\mathrm{C}_{\mathrm{ab}} / 100\right)$ \\
\hline $\mathbf{5}$ & $\mathrm{TC}_{\mathrm{ar}}$ & $\exp \left(-\mathrm{C}_{\mathrm{ar}} / 100\right)$ \\
\hline $\mathbf{6}$ & $\mathrm{TC}_{\mathrm{w}}$ & $\exp \left(-50 \mathrm{C}_{\mathrm{w}}\right)$ \\
\hline $\mathbf{7}$ & $\mathrm{TC}_{\mathrm{dm}}$ & $\exp \left(-100 \mathrm{C}_{\mathrm{dm}}\right)$ \\
\hline
\end{tabular}


Table 4. Upper and lower bounds for the state vector terms (in transformed space, where appropriate) used in the simulations, along with the temporal trajectory assumed.

\begin{tabular}{|l|l|l|l|l|}
\hline$\#$ & Symbol & Lower limit & Upper limit & Temporal function \\
\hline $\mathbf{1}$ & $\mathrm{TLAI}$ & 0.067 & 0.995 & $\mathrm{LAI}=0.21+3.51 \sin ^{5}(\pi \mathrm{t})$ \\
\hline $\mathbf{4}$ & $\mathrm{TC}_{\mathrm{ab}}$ & 0.135 & 1.0 & $\begin{array}{l}\mathrm{C}_{\mathrm{ab}}=10.5+208.7 \mathrm{t}: \mathrm{t}<=0.5 \\
\mathrm{C}_{\mathrm{ab}}=219.2-208.7 \mathrm{t}: \mathrm{t}>=0.5\end{array}$ \\
\hline $\mathbf{6}$ & $\mathrm{TC}_{\mathrm{w}}$ & 0.135 & 1.0 & $\mathrm{C}_{\mathrm{w}}=0.068+$ \\
\hline $\mathbf{7}$ & $\mathrm{TC}_{\mathrm{dm}}$ & 0.135 & & $0.020\left(\sin (\pi \mathrm{t}+0.1)^{*} \sin (6 \pi \mathrm{t}+0.1)\right)$ \\
\hline $\mathbf{8}$ & $\mathrm{N}$ & 1 & 1.0 & $\mathrm{C}_{\mathrm{dm}}=0.01$ \\
\hline $\mathbf{9}$ & $s_{1}$ & 0.001 & 2.5 & $\mathrm{~N}=1$ \\
\hline
\end{tabular}


Table 5. Model uncertainty $\gamma$ for each parameter, calculated from the synthetic model state vector. $T C_{d m}$ and $\mathrm{N}$ were kept constant, so have no theoretical model uncertainty associated.

\begin{tabular}{|l|l|l|l|}
\hline$\#$ & Symbol & First difference & Second \\
& & uncertainty & difference \\
uncertainty
\end{tabular}


Table 6: Mean posterior uncertainty. Figures refer to the complete daily time series, while figures in brackets refer to the mean posterior uncertainty only considering the dates where observations are available.

1365

\begin{tabular}{|l|c|l|l|l|l|l|}
\hline & \multicolumn{2}{|l|}{ Non-cloudy } & \multicolumn{2}{l|}{ Cloudy } \\
\hline Symbol & $\begin{array}{l}\text { Uncertainty } \\
\text { Single Obs. }\end{array}$ & $\begin{array}{l}\text { Uncertainty } \\
1^{\text {st }} \text { Diff }\end{array}$ & $\begin{array}{l}\text { Uncertainty } \\
2^{\text {nd }} \text { Diff }\end{array}$ & $\begin{array}{l}\text { Uncertainty } \\
\text { Single Obs. }\end{array}$ & $\begin{array}{l}\text { Uncertainty } \\
1^{\text {st }} \text { Diff }\end{array}$ & $\begin{array}{l}\text { Uncertainty } \\
2^{\text {nd }} \text { Diff }\end{array}$ \\
\hline $\mathrm{TLAI}$ & $0.18(0.05)$ & $0.04(0.04)$ & $0.06(0.06)$ & $0.21(0.05)$ & $0.06(0.05)$ & $0.09(0.07)$ \\
\hline $\mathrm{TC}_{\mathrm{ab}}$ & $0.20(0.10)$ & $0.04(0.04)$ & $0.06(0.06)$ & $0.22(0.09)$ & $0.06(0.05)$ & $0.08(0.06)$ \\
\hline $\mathrm{TC}_{\mathrm{w}}$ & $0.23(0.18)$ & $0.07(0.07)$ & $0.13(0.13)$ & $0.24(0.19)$ & $0.10(0.10)$ & $0.17(0.16)$ \\
\hline $\mathrm{TC}_{\mathrm{dm}}$ & $0.24(0.22)$ & $0.13(0.13)$ & $0.28(0.28)$ & $0.24(0.23)$ & $0.19(0.19)$ & $0.36(0.35)$ \\
\hline$N$ & $0.29(0.55)$ & $0.21(0.21)$ & $0.37(0.37)$ & $0.27(0.55)$ & $0.32(0.32)$ & $0.44(0.40)$ \\
\hline$s_{1}$ & $0.17(0.04)$ & $0.02(0.02)$ & $0.03(0.03)$ & $0.20(0.04)$ & $0.04(0.03)$ & $0.05(0.03)$ \\
\hline
\end{tabular}

1366

Table 7: Single observation posterior correlation matrix. Elements above the main diagonal show the results for DoY 186, whereas the elements below the main diagonal represent the median of all dates.

\begin{tabular}{|l|l|l|l|l|l|l|}
\hline Symbol & $\mathrm{TLAI}$ & $\mathrm{TC}_{\mathrm{ab}}$ & $\mathrm{TC}_{\mathrm{w}}$ & $\mathrm{TC}_{\mathrm{dm}}$ & $N$ & $s_{1}$ \\
\hline TLAI & 1.00 & 0.16 & -0.05 & 0.47 & -0.25 & 0.58 \\
\hline $\mathrm{TC}_{\mathrm{ab}}$ & -0.44 & 1.00 & 0.15 & -0.11 & -0.47 & 0.34 \\
\hline $\mathrm{TC}_{\mathrm{w}}$ & -0.42 & 0.35 & 1.00 & 0.04 & 0.01 & -0.14 \\
\hline $\mathrm{TC}_{\mathrm{dm}}$ & 0.30 & 0.27 & -0.27 & 1.00 & 0.42 & -0.36 \\
\hline$N$ & 0.00 & -0.21 & 0.07 & -0.43 & 1.00 & -0.85 \\
\hline$s_{1}$ & 0.76 & -0.53 & -0.40 & -0.25 & -0.28 & 1.00 \\
\hline
\end{tabular}


Table 8: Uncertainty reduction relative to the single observation inversion, as well as percentage of cases where the true parameter lies within the estimated 95\% confidence interval. Results for non-cloudy scenario are reported. both complete time series

\begin{tabular}{|l|l|l|l|l|l|l|l|l|l|l|}
\hline & \multicolumn{9}{|l|}{ Complete time series } & \multicolumn{6}{l|}{ Observations only } \\
\hline$\#$ & Symbol & $\begin{array}{l}\text { Unc. } \\
\text { red } \\
1^{\text {st }} \\
\text { diff }\end{array}$ & $\begin{array}{l}\text { Unc. } \\
\text { red. } \\
2^{\text {nd }} \\
\text { diff }\end{array}$ & $\begin{array}{l}\% \text { cases } \\
\left(1^{\text {st }} \text { diff }\right)\end{array}$ & $\begin{array}{l}\% \text { cases } \\
\left(2^{\text {nd }} \text { diff }\right)\end{array}$ & $\begin{array}{l}\text { Unc. } \\
\text { red } \\
1^{\text {st }} \\
\text { diff }\end{array}$ & $\begin{array}{l}\text { Unc. } \\
\text { red. } \\
2^{\text {nd }} \\
\text { diff }\end{array}$ & $\begin{array}{l}\% \text { cases } \\
\left(1^{\text {st }} \text { diff }\right.\end{array}$ & $\begin{array}{l}\% \text { cases } \\
\left(2^{\text {nd }} \text { diff }\right)\end{array}$ & $\begin{array}{l}\% \text { cases } \\
(\text { single })\end{array}$ \\
\hline 1 & $\mathrm{TLAI}_{4}$ & 4.89 & 2.96 & 75.3 & 90.4 & 1.44 & 0.85 & 72.6 & 91.8 & 63.0 \\
\hline 4 & $\mathrm{TC}_{\mathrm{ab}}$ & 5.24 & 3.58 & 61.1 & 65.2 & 2.57 & 1.74 & 60.3 & 65.8 & 65.8 \\
\hline 6 & $\mathrm{TC}_{\mathrm{w}}$ & 3.47 & 1.77 & 51.2 & 69.9 & 2.72 & 1.38 & 50.7 & 71.2 & 60.3 \\
\hline 7 & $\mathrm{TC}_{\mathrm{dm}}$ & 1.82 & 0.85 & 87.7 & 100.0 & 1.64 & 0.76 & 87.7 & 100.0 & 57.5 \\
\hline 8 & $N$ & 1.40 & 0.79 & 59.2 & 100.0 & 2.67 & 1.50 & 58.9 & 100.0 & 60.3 \\
\hline 9 & $s_{1}$ & 7.59 & 6.43 & 67.1 & 72.3 & 2.13 & 1.56 & 63.0 & 72.6 & 75.3 \\
\hline & Mean & 4.07 & 2.73 & 66.9 & 83.0 & 2.20 & 1.30 & 65.5 & 83.6 & 63.7 \\
\hline
\end{tabular}


Table 9: Uncertainty reduction relative to the single observation, as well as percentage of cases where the true parameter lies within the estimated 95\% confidence interval. Results for cloudy scenario.

\begin{tabular}{|l|l|l|l|l|l|l|l|l|l|l|}
\hline & \multicolumn{9}{|l|}{ Complete time series } & \multicolumn{6}{l|}{ Observations only } \\
\hline$\#$ & Symbol & $\begin{array}{l}\text { Unc. } \\
\text { red } \\
1^{\text {st }} \\
\text { diff }\end{array}$ & $\begin{array}{l}\text { Unc. } \\
\text { red. } \\
2^{\text {nd }} \\
\text { diff }\end{array}$ & $\begin{array}{l}\% \text { cases } \\
\left(1^{\text {st }} \text { diff }\right)\end{array}$ & $\begin{array}{l}\% \text { cases } \\
\left(2^{\text {nd }} \text { diff }\right)\end{array}$ & $\begin{array}{l}\text { Unc. } \\
\text { red } \\
1^{\text {st }} \\
\text { diff }\end{array}$ & $\begin{array}{l}\text { Unc. } \\
\text { red. } \\
2^{\text {nd }} \\
\text { diff }\end{array}$ & $\begin{array}{l}\% \text { cases } \\
\left(1^{\text {st }} \text { diff }\right)\end{array}$ & $\begin{array}{l}\% \text { cases } \\
\left(2^{\text {nd }} \text { diff }\right)\end{array}$ & $\begin{array}{l}\% \text { cases } \\
(\text { single })\end{array}$ \\
\hline 1 & $\mathrm{TLAI}$ & 3.33 & 2.39 & 82.7 & 74 & 0.965 & 0.68 & 88.9 & 91.7 & 63.9 \\
\hline 4 & $\mathrm{TC}_{\mathrm{ab}}$ & 3.68 & 2.93 & 80.0 & 89.6 & 1.82 & 1.58 & 61.1 & 83.3 & 58.3 \\
\hline 6 & $\mathrm{TC}_{\mathrm{w}}$ & 2.33 & 1.4 & 64.1 & 85.2 & 1.90 & 1.18 & 83.3 & 88.9 & 61.1 \\
\hline 7 & $\mathrm{TC}_{\mathrm{dm}}$ & 1.25 & 0.669 & 100 & 100 & 1.18 & 0.656 & 100 & 100 & 58.3 \\
\hline 8 & $N$ & 0.835 & 0.597 & 91 & 100 & 1.73 & 1.38 & 88.9 & 100 & 58.3 \\
\hline 9 & $s_{1}$ & 4.65 & 4.53 & 63.6 & 78.1 & 1.57 & 1.35 & 69.4 & 72.2 & 75.0 \\
\hline & Mean & 2.68 & 2.09 & 80.2 & 87.8 & 1.53 & 1.14 & 82.0 & 89.3 & 62.5 \\
\hline
\end{tabular}




\section{Figures}

Figure 1. Base level state vector estimated from inverting single observations, (left column) and for model uncertainty unknown and estimated through cross-validation-first difference constraint (central column) and second difference constraint (third column). Results for each of the six parameters are shown in rows. True values are shown as a dashed line. The full lines are the posterior means, and the shaded area represents the associated +/-1.96 standard deviations interval. MSI observations are shown as open symbols. Crosses along the bottom of the third row indicate the location of the cross validation acquisition dates. 

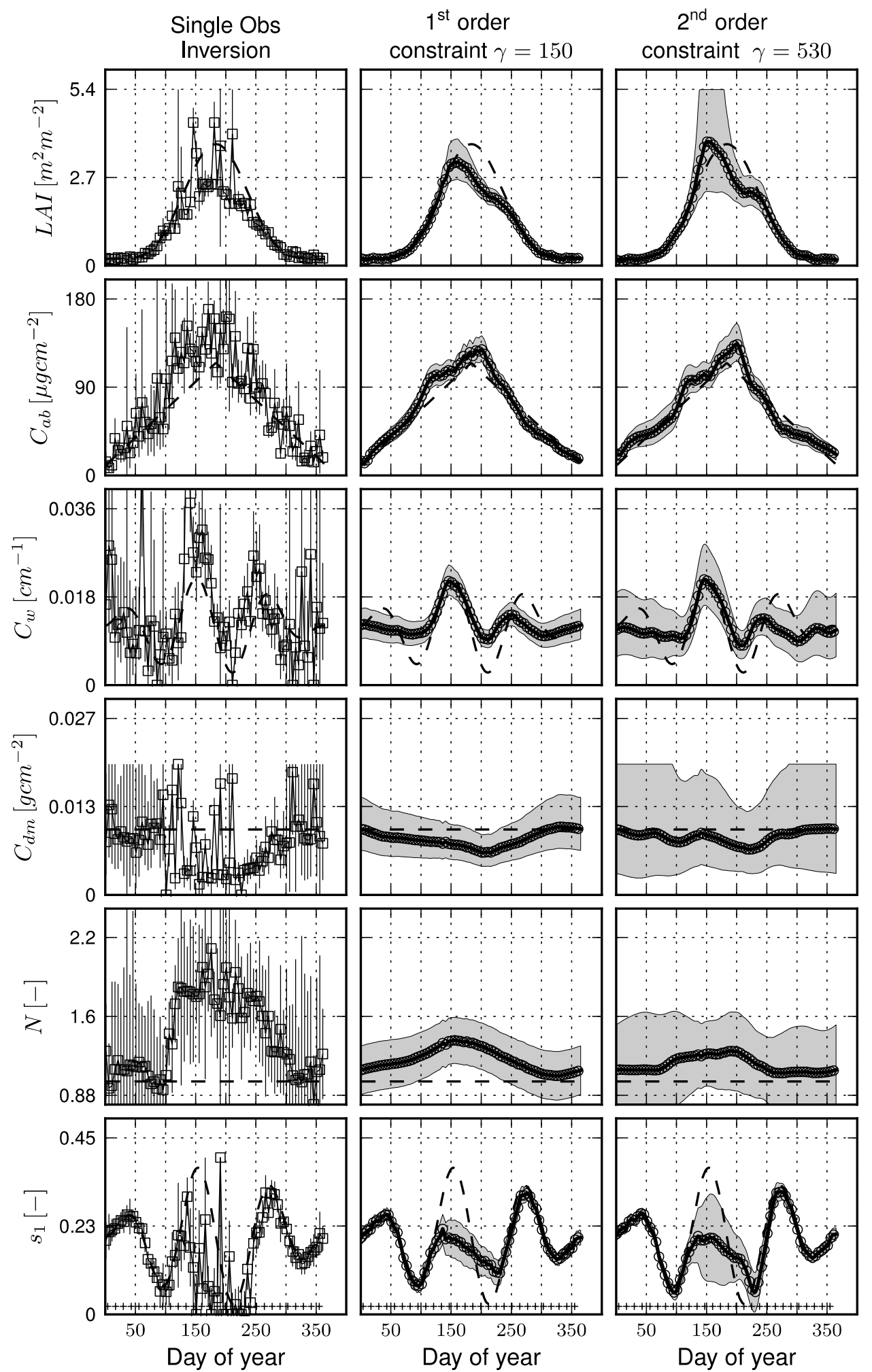
1387 Figure 2. Error in cross validation scaled by observational uncertainty for varying model 1388 uncertainty $\gamma$ for first and second order constraints. Vertical lines around 200 represent the 1389 theoretical value of $\gamma$ for each of the 4 time-varying state variables using a first order 1390 constraint, and vertical lines around 5000 represent the theoretical values for $\gamma$ for each of the 13914 time-varying state variables using second order constraint.

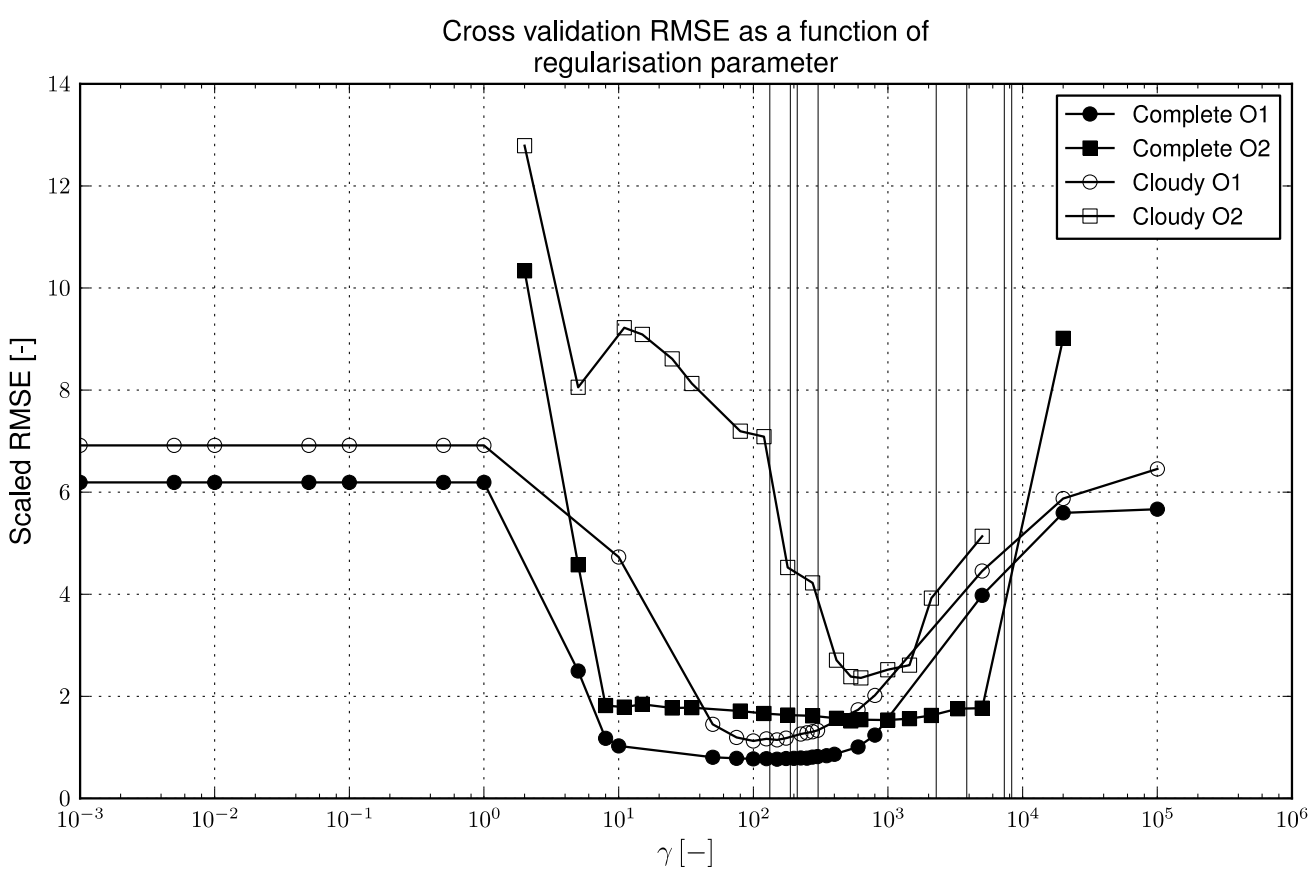


Figure 3. Base level state vector estimated from inverting single observations, (left column) and for model uncertainty unknown and estimated through cross-validation-first difference constraint (central column) and second difference constraint (third column). Reduced number of acquisitions due to cloud cover scenario. Results for each of the six parameters are shown in rows. True values are shown as a dashed line. The full lines are the posterior means, and the shaded area represents the associated +/-1.96 standard deviations interval. MSI observations are shown as open symbols. Crosses along the bottom of the third row indicate the location of the cross validation acquisition dates. 

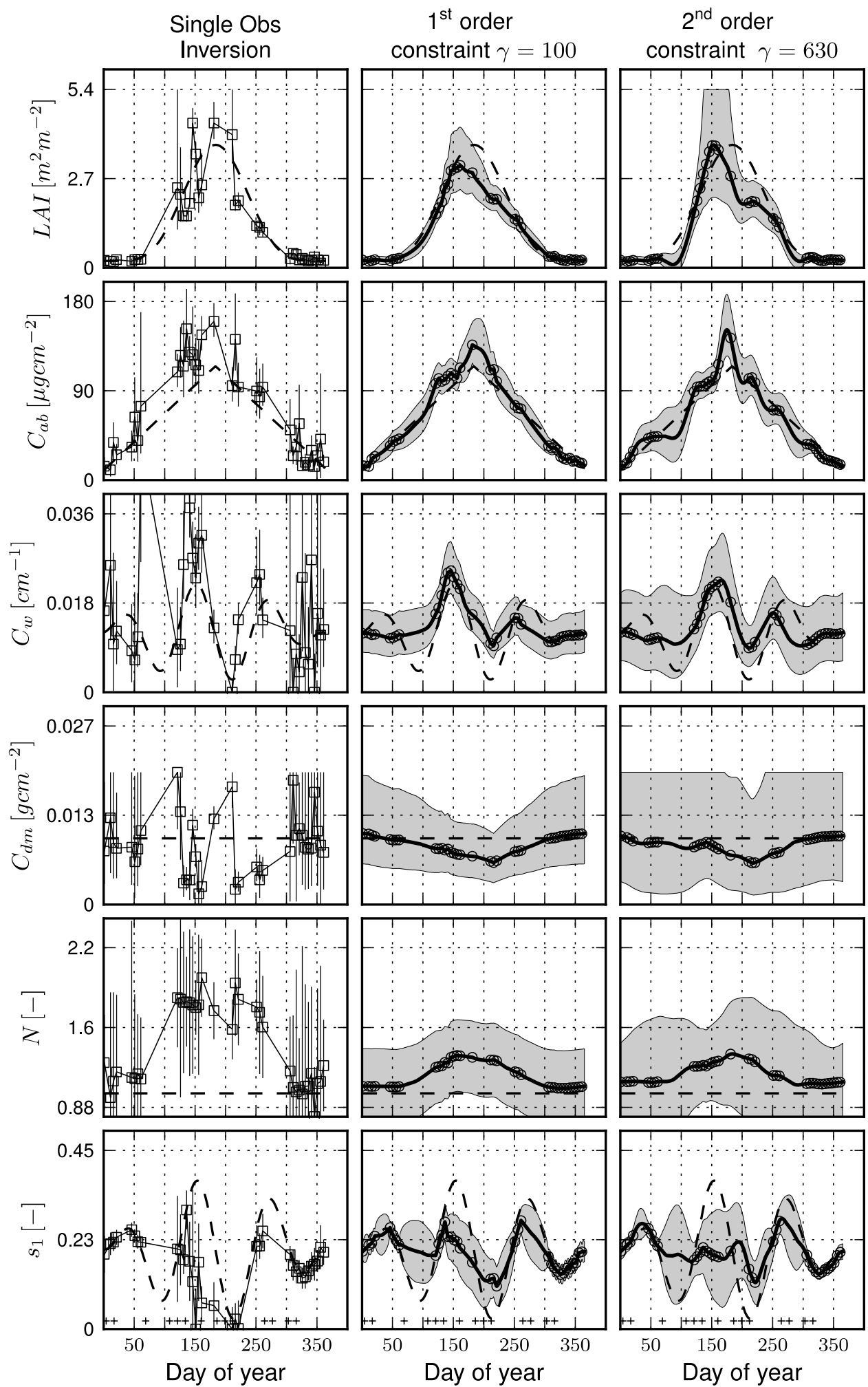

Figure 4. Posterior correlation matrices for the cloudy scenario. Labels indicate the location of the first day for component of the state vector. 

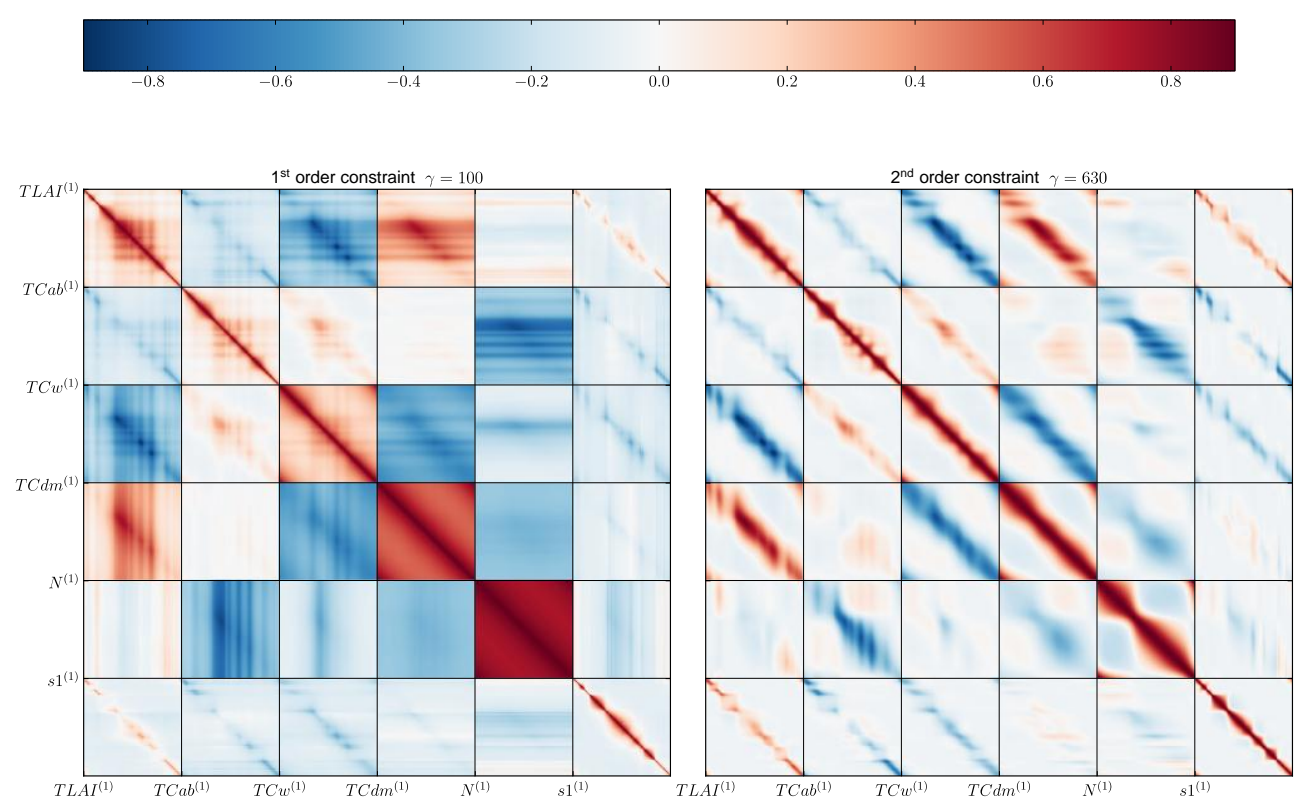

1397

1398 